\title{
1 Quantitative in vivo analyses reveal a complex pharmacogenomic landscape in
}

7 Authors:

8 Chuan Li ${ }^{1,9}$, Wen-Yang Lin ${ }^{2,9}$, Hira Rizvi ${ }^{3}$, Hongchen Cai ${ }^{2}$, Christopher D. McFarland ${ }^{1}$, Zoë N.

9 Rogers $^{2}$, Maryam Yousefi ${ }^{2}$, Ian P. Winters ${ }^{2}$, Charles M. Rudin ${ }^{4,5}$, Dmitri A. Petrov ${ }^{1,6^{*}}$ and Monte

10 M. Winslow 2,6,7,8*

\section{Affiliations:}

$12{ }^{1}$ Department of Biology, Stanford University, Stanford, CA, USA

$13{ }^{2}$ Department of Genetics, Stanford University School of Medicine, Stanford, CA, USA

$14{ }^{3}$ Druckenmiller Center for Lung Cancer Research, Memorial Sloan Kettering Cancer Center, New York, USA

$15{ }^{4}$ Department of Medicine, Thoracic Oncology Service, Memorial Sloan Kettering Cancer Center, New York, USA

$16{ }^{5}$ Department of Medicine, Weill Cornell Medical College, New York, USA

$17{ }^{6}$ Cancer Biology Program, Stanford University School of Medicine, Stanford, CA, USA

$18{ }^{7}$ Department of Pathology, Stanford University School of Medicine, Stanford, CA, USA

$19{ }^{8}$ Stanford Cancer Institute, Stanford University School of Medicine, Stanford, CA, USA

$20 \quad{ }^{9}$ These authors contributed equally

$22 *$ Corresponding authors:

23 Monte M. Winslow, Stanford University School of Medicine | 279 Campus Drive, Beckman Center, Room B256, Stanford, CA

2494305. Phone: 650-725-8696|Fax: 650-725-1534|E-mail: mwinslow@ stanford.edu

27 Phone: 650-736-1169|Fax: 650-723-6132|E-mail: dpetrov@ stanford.edu 


\section{Abstract:}

32 The lack of knowledge about the relationship between tumor genotypes and therapeutic

33 responses remains one of the most important gaps in enabling the effective use of cancer

34 therapies. Here, we couple a multiplexed and quantitative platform with robust statistical

35 methods to enable pharmacogenomic mapping of lung cancer treatment responses in vivo. We

36 uncover a surprisingly complex map of genotype-specific therapeutic responses, with over $20 \%$

37 of possible interactions showing significant resistance or sensitivity. We validate one of these

38 interactions - the resistance of Keap1 mutant tumors to platinum therapy - using a large patient

39 response dataset. Our results highlight the importance of understanding the genetic determinants

40 of treatment responses in the development of precision therapies and define a strategy to identify

41 such determinants.

\section{Main Text:}

Efforts over the past decade have generated many novel cancer therapies. However, the

45 lack of understanding of the relationship between tumor genotype and therapeutic response

46 remains a major challenge in translating these therapies into precision cancer treatments ${ }^{1}$. While

47 the genetic complexity of human cancer could in principle thwart therapeutic efforts that

48 disregard biomarker-driven patient selection, it remains unclear the extent to which tumor

49 suppressor gene alterations impact therapeutic responses ${ }^{2}$. Precisely mapping the

50 pharmacogenomic landscape should help design better clinical trials and select more effective

51 therapies for individual patients.

52 The pharmacogenomic landscape of cancer drug responses has been investigated using

53 cell lines, patient-derived xenografts (PDXs), and patients themselves ${ }^{3-6}$. However, cell lines 
54 grown in vitro lack the appropriate in vivo environment and the genotypic heterogeneity of

55 patient tumors. PDXs and human cell line transplantation models recapitulate some aspects of in

56 vivo growth, but growth factor/receptor incompatibility, growth in non-orthotopic sites, and the

57 obligate absence of the adaptive immune system compromise these approaches ${ }^{7-9}$. Furthermore,

58 human tumor-derived systems almost invariably have large numbers of mutations and genomic

59 rearrangements, and thus even large-scale analyses often lack the statistical power to glean

60 cause-and-effect relationships between individual genetic alterations and therapeutic responses

$61 \quad 3,10$.

62 Genetically engineered mouse models of cancer uniquely enable the introduction of

63 defined genetic alterations into adult somatic cells, which leads to the generation of

64 autochthonous tumors ${ }^{11}$. These tumors can recapitulate the genomic alterations, gene expression

65 state, histopathology, and therapy-refractive nature of corresponding human cancers ${ }^{12,13}$. Despite

66 the potential value of these models in pre-clinical translation studies, the breadth of their utility

67 has been limited by the fact that they are neither readily scalable nor sufficiently quantitative ${ }^{14}$.

68 To increase the scope and precision of in vivo cancer modeling and to assess tumor

69 suppressor gene function in a multiplexed manner, we recently developed a method based on

70 tumor-barcoding coupled with high-throughput barcode sequencing (Tuba-seq). This method

71 integrates CRISPR/Cas9-based somatic genome engineering and molecular barcoding into well-

72 established Cre/Lox-based genetically engineered mouse models of oncogenic Kras-driven lung

73 cancer ${ }^{14}$. The initiation of lung tumors with pools of barcoded Lenti-sgRNA/Cre viral vectors

74 enables the generation of many tumors of different genotypes in parallel. All neoplastic cells

75 within each clonal tumor have the same two-component barcode, where each sgID region

76 identifies the sgRNA and the random barcode (BC) is unique to each tumor. Subsequent high- 
77 throughput sequencing of the sgID-BC region from bulk tumor-bearing lungs can quantify the

78 number of neoplastic cells in each tumor of each genotype.

$$
\text { We initiated lung tumors in } \operatorname{Kras}^{L S L-G 12 D /+} ; \operatorname{Rosa}^{L S 6^{L L-T o m a t o}} ; H 11^{\text {LSL-Cas9 }}\left(K T ; H 11^{\text {LSL-Cas9 }}\right)
$$

80 mice and control Cas9-negative $K T$ mice with a pool of barcoded Lenti-sgRNA/Cre vectors

81 targeting eleven putative tumor suppressors and four control vectors with inert sgRNAs (Lenti-

$82 \operatorname{sg} T S^{\text {Pool} / C r e ; ~ F i g . ~ 1 a) . ~ T u m o r ~ s u p p r e s s o r s ~ w e r e ~ s e l e c t e d ~ b a s e d ~ o n ~ c o m m o n ~ o c c u r r e n c e ~ i n ~ h u m a n ~}$

83 lung adenocarcinomas and previously suggested roles in oncogenesis ${ }^{15} .18$ weeks after tumor

84 initiation, the sgID-BC region from each bulk tumor-bearing lung was PCR amplified and

85 sequenced to quantify tumor sizes (Fig. 1a).

We optimized our Tuba-seq experimental protocols and analysis pipeline to eliminate

87 sgRNA-sgID/barcode uncoupling due to lentiviral template switching and to minimize PCR,

88 sequencing, and clustering errors (Fig. 1b, Supplementary Fig. 1a, and Methods) ${ }^{15}$. Our new

89 streamlined pipeline essentially eliminated the impact of read errors, as assessed by multiple

90 metrics, including the remaining spurious tumors from spike-in barcodes with known sequences

91 and correspondence of tumor barcodes with those from the lentiviral plasmid pool (Fig. 1c, d,

92 Supplementary Fig. 1b). Quantification of the impact of tumor suppressor gene inactivation on

93 tumor growth in $K T ; H 11^{L S L-C a s 9}$ mice using our optimized method uncovered effects that were

94 generally consistent with our previous analyses but with greater magnitudes of tumor

95 suppression (Fig. 1e; Supplementary Fig. 1c, d and 2, sign test for differences in magnitudes, $P$

$96=0.001)^{14}$. Consistent with the robustness of our methods, analysis of the $K T$ mice with Lenti-

$97 \mathrm{sg} T S^{P o o l} / \mathrm{Cre}$-initiated tumor revealed no false-positive tumor suppressive effects

98 (Supplementary Fig. 1d, e). 
When quantifying tumor suppressor gene effects using Tuba-seq, each mouse represents

100

101

102

103

104

105

106

107

108

109

110

111

112

113

114

115

116

117

118

119

120

121

an internally controlled experiment in which tumor size metrics can be normalized to tumors

with inert sgRNAs within the same animal (Fig. 1e, Supplementary Fig. 1) ${ }^{15}$. In contrast, comparing tumor size distributions among groups of mice, such as between untreated and drugtreated groups, requires methods that overcome the technical and biological differences between mice. To address these challenges, we rigorously modeled drug responses and genotype-specific responses under the assumption that while tumors of all sizes respond equally to each treatment, the treatment effect can vary by genotype. Specifically, we estimated the drug effect on control tumors (those with inert sgRNAs) and then applied this effect to all tumors to calculate an expected distribution of tumor sizes after treatment (Fig. 2a and Methods). Genotype-specific therapeutic responses (GSTRs) were quantified by comparing the observed distribution of tumor sizes for tumors of a certain genotype after treatment with the expected distribution derived from the untreated mice. We developed two statistics to characterize GSTRs, the first is based on the relative numbers of tumors above a certain size after treatment (ScoreRTN - Relative Tumor Number) and the second compares the geometric mean of tumors from the full distribution of tumor sizes (ScoreRGM - Relative Geometric Mean) (Methods). By assessing the performance of the two statistics, we showed that both statistics are unbiased and have substantial and similar power although one statistic may outperform the other if the genotype-specific response is not uniform across tumor sizes (Methods, Fig. 2b-c and Supplementary Fig. 3, 4 and 5).

We applied Tuba-seq and our statistical metrics to assess the genotype-specific therapeutic responses of 11 genotypes of lung tumors to a panel of eight single and combination therapies (Fig. 1a and 2d). These therapies were chosen to perturb diverse signaling pathways and assess the genotype-dependency of chemotherapy responses. KT;H1 $1^{L S L-C a s 9}$ mice with 
122 Lenti-sgTS ${ }^{\text {Pool}} / \mathrm{Cre}$-initiated lung tumors were treated for three weeks with one of the eight

123 therapies followed by Tuba-seq analysis (Fig. 1a). The total cancer cell numbers estimated by

124 Tuba-seq were highly correlated with total tumor-bearing lung weights, which varied

125 substantially among mice even within the same groups (Supplementary Fig. 6a-c). Despite such

126 mouse-to-mouse variation, analysis of the overall tumor burden and the number of tumors with

127 inert sgRNAs identified significant overall effects of five treatments (Supplementary Fig. 6).

128 We compared the tumor size profiles of treated mice with those of untreated mice and

129 calculated the ScoreRTN and ScoreRGM (Supplementary Fig. 7). For both statistics, we

130 estimated the magnitudes of GSTR and the associated $P$-values. Across all genotypes and

131 treatments, the two statistics were well-correlated in magnitude as expected under the model of

132 proportional tumor responses (Supplementary Fig. $7 \mathbf{b} ; r=0.86, P=10^{-46}$ ). Among the 88

133 assessed genotype-treatment pairs, 20 and 17 significant GSTRs $(P<0.05)$ were identified by

134 ScoreRTN and ScoreRGM, respectively. Of these, 19 genotype-treatment interactions were

135 significant by one statistic $(P<0.05)$ and at least marginally significant $(P<0.1)$ by the other

136 (Supplementary Fig. 7a, b; Table S1). We derived a composite measure of GSTR $(\widehat{G})$ with the 137 magnitude estimated from the inverse variance weighted average of the two statistics (Methods,

138 Fig. 2f). Analysis of genotype-specific effects across treatments highlighted similarities among

139 tumor suppressors, including those of Lkb1 and Setd2 known to have redundant tumor

140 suppressive effects ${ }^{5}$, while combination treatments clustered with their corresponding single

141 therapies (Supplementary Fig. 7c, d). Power analysis showed that our findings were robust to

142 the choice of inert sgRNAs, cancer cell number cutoff, and inaccurate estimation of drug effect

\section{3 (Supplementary Fig. 8, 9).}


145 tumors to the CDK4/6 inhibitor, palbociclib. This resistance is consistent with the biochemical

146 features of this pathway and clinical findings in breast cancer and hepatocellular carcinoma ${ }^{16-18}$

147 (Supplementary Fig. 11f). Our ability to rediscover this interaction serves as a positive control

148 of our method and is consistent with the expectation that some pharmacogenomic interactions

149 transcend cancer types.

150 To further test the performance of our experimental and statistical procedures, we

151 performed two additional experiments. First, as a negative control for GSTR identification, we

152 treated Cas9-negative $K T$ mice with a combination of chemotherapy and Mek-inhibition

153 (Supplementary Fig. 10a). This treatment led to a dramatic reduction in tumor sizes compared

154 to untreated $K T$ mice (Supplementary Fig. 10b). Only one false positive GSTR was identified

155 (ScoreRTN, $\mathrm{P}=0.03 ;$ ScoreRGM, P = 0.07) with a very weak magnitude of the effect $(\widehat{G}=0.093$,

156 while the minimum magnitude of significant GSTR in the main experiment was 0.108; Fig. 2g).

157 Combined with the fact that none of the individual inert sgRNAs (sgNeo1, sgNeo2, sgNeo3, and

$158 \mathrm{sgNT}$ ) had significant effects by either metric for any of the treatments in our main

159 pharmacogenomic mapping experiment, this experiment provided additional confidence in the

160 veracity of the detected GSTRs (Fig. 2f, g).

161 Simulation suggests that these cohort sizes have substantial power (Supplementary Fig.

162 4); therefore, we next attempted to rediscover the genotype-palbociclib interactions. We initiated

163 tumors in a similar, albeit somewhat smaller cohort of $K T ; H 11^{\text {LSL-Cas9 }}$ mice with Lenti-

$164 \mathrm{sg} T S^{\text {Pool}} / \mathrm{Cre}$ and repeated the palbociclib-treatment. Analyses of these mice again identified $R b 1$

165 inactivation as a mediator of palbociclib resistance (Fig. 2f, Supplementary Fig. 10). Smad4-

166 deficient tumors, which showed modest resistance in our initial experiment, showed nominal 
167 resistance in the repeat experiment $(\hat{G}=0.167)$, although this interaction was not significant $(P=0.17$ and 0.20 for ScoreRTN and ScoreRGM, respectively).

While the positive and negative predictive values of cancer cell line studies are often

170 questioned ${ }^{19}$, the scale at which these in vitro studies can be performed has enabled the

171 generation of drug response data across large panels of cell lines ${ }^{4,20}$. We compared our findings

172 to the largest dataset of cell line-therapeutic responses (Genomics of Drug Sensitivity in Cancer;

173 GDSC) $)^{3}$ in which all five of our monotherapies were assessed (paclitaxel, palbociclib,

174 phenformin, everolimus/rapamycin, and trametinib) ${ }^{3}$. Among the genotype-treatment pairs

175 assessed in both studies, nine had significant effects in our analysis, but only one of these

176 genotype-treatment interactions was significant in GDSC (Rb1-palbociclib)(Fig. 2i and

177 Supplementary Fig 12). Three of the significant genotype-specific resistances, which were only

178 uncovered in vivo, involved Smad4-deficiency (Fig. 2i). Given the absence of microenvironment

179 stimulation of the TGF $\beta / \mathrm{Smad} 4$ pathway in culture, these findings suggest that targeting

180 paracrine TGF $\beta$ signaling could reduce the efficacy of cancer therapies in some contexts ${ }^{21}$.

181 Although most of the detected pharmacogenomic interactions we uncovered are novel,

182 several lines of evidence derived from clinical and preclinical data are consistent with our

183 observations. For instance, $L k b l$-inactivation reduced sensitivity to mTOR inhibition in our data,

184 which is supported by previous anecdotal data from the analysis of lung adenocarcinoma patient-

185 derived primary cultures in vitro (Supplementary Fig. 13) ${ }^{5}$. The ultimate goal of our study is to

186 find genotype-treatment responses that predict lung adenocarcinoma patient responses. Lung

187 adenocarcinoma patients are often treated with first-line platinum-containing combination

188 therapies. In our analysis, Keapl-inactivation specifically led to resistance to treatments that

189 included carboplatin, while not promoting significant resistance to the other therapies (Fig. 2f). 
190 To investigate the clinical impact of tumor suppressor genotype on lung adenocarcinoma

191 responses, we queried the tumor suppressor genotypes and therapeutic benefit of platinum-

192 containing treatments (assessed as time-to-next-treatment) of 216 oncogenic $K R A S$-driven

193 human lung adenocarcinoma patients treated at Memorial Sloan Kettering Cancer Center

194 (Methods). When each gene was assessed individually, both KEAPI and LKB1 mutations were

195 associated with worse clinical outcomes $\left(P=6 \times 10^{-6}\right.$, Fig 2 j and $P=0.06$, Supplementary Fig.

$196 \mathbf{1 4 j}$, respectively). However, the marginally significant effect of $L K B 1$ mutation appears to be

197 driven by the co-occurrence of KEAP1 mutations with LKB1 mutations (Supplementary Fig.

198 14). This is also well supported by our pharmacogenomic data in which $L k b l$-inactivation did

199 not confer resistance to platinum-containing treatments (Fig. 2f). We further quantified the

200 hazard ratio of the mutational status of the 11 genes in a manner that takes into account the effect

201 of other co-incident mutations. This analysis confirmed that mutation of KEAP1 correlated with

202 a shorter time-to-next-treatment, which is consistent with our Tuba-seq results as well as a

203 previous study on the impact of KEAP1/NRF2-pathway alterations on platinum responses (Fig.

$2042 \mathbf{j}, \mathbf{k})^{22,23}$. Our in vivo pharmacogenomic platform, in which the responses of tumors with

205 defined genotypes can be quantified, establishes direct causal relationships between genotype

206 and treatment responses and enables accurate interpretation of patient data.

207 We uncovered a surprisingly complex pharmacogenomic map of resistance and

208 sensitivity of $K R A S$-driven lung adenocarcinoma. Across diverse tumor suppressor genotypes

209 and treatments, genotype-treatment interactions were very common ( $20 \%$ in our study),

210 suggesting that genetic differences among tumors may underlie much of the heterogeneity in

211 treatment responses. Our study required only a small number of animals, thus highlighting the

212 power of our platform in confirming existing, and prioritizing new, genotype-specific drug 
214 additional therapies (and combination therapies) across a greater diversity of cancer genotypes

215 will provide a cause-and-effect pharmacogenomic framework from which novel biological

216 hypotheses and precision treatment approaches will emerge.

\section{References:}

2191 Roychowdhury, S. \& Chinnaiyan, A. M. Translating genomics for precision cancer medicine. Annu Rev Genomics Hum Genet 15, 395-415, doi:10.1146/annurev-genom090413-025552 (2014). van de Haar, J. et al. Identifying Epistasis in Cancer Genomes: A Delicate Affair. Cell 177, 1375-1383 (2019).

3 Iorio, F. et al. A Landscape of Pharmacogenomic Interactions in Cancer. Cell 166, 740754, doi:10.1016/j.cell.2016.06.017 (2016).

4 Barretina, J. et al. The Cancer Cell Line Encyclopedia enables predictive modelling of anticancer drug sensitivity. Nature 483, 603-607, doi:10.1038/nature11003 (2012). Lee, J. K. et al. Pharmacogenomic landscape of patient-derived tumor cells informs precision oncology therapy. Nat Genet 50, 1399-1411, doi:10.1038/s41588-018-0209-6 (2018).

2316 Roper, N., Stensland, K. D., Hendricks, R. \& Galsky, M. D. The landscape of precision cancer medicine clinical trials in the United States. Cancer Treat Rev 41, 385-390, doi:10.1016/j.ctrv.2015.02.009 (2015). Translational Cancer Research. Cancer discovery 4, 998-1013, doi:10.1158/21598290.CD-14-0001 (2014). Ben-David, U. et al. Patient-derived xenografts undergo mouse-specific tumor evolution.

8 Ben-David, U. et al. Patient-derived xenografts undergo

9 Hoffman, R. M. J. N. R. C. Patient-derived orthotopic xenografts: better mimic of metastasis than subcutaneous xenografts. 15, 451 (2015).

10 Gao, H. et al. High-throughput screening using patient-derived tumor xenografts to predict clinical trial drug response. 21, 1318 (2015).

11 Kersten, K., de Visser, K. E., van Miltenburg, M. H. \& Jonkers, J. Genetically engineered mouse models in oncology research and cancer medicine. EMBO Mol Med 9, 137-153, doi:10.15252/emmm.201606857 (2017). closing the gap between preclinical data and trial outcomes. 72, 2695-2700 (2012).

13 Chen, Z. et al. A murine lung cancer co-clinical trial identifies genetic modifiers of therapeutic response. Nature 483, 613-617, doi:10.1038/nature10937 (2012). 
25014 Rogers, Z. N. et al. Mapping the in vivo fitness landscape of lung adenocarcinoma tumor suppression in mice. Nat Genet 50, 483-486, doi:10.1038/s41588-018-0083-2 (2018). Rogers, Z. N. et al. A quantitative and multiplexed approach to uncover the fitness landscape of tumor suppression in vivo. Nat Methods 14, 737-742, doi:10.1038/nmeth.4297 (2017).

16 Bollard, J. et al. Palbociclib (PD-0332991), a selective CDK4/6 inhibitor, restricts tumour
growth in preclinical models of hepatocellular carcinoma. Gut 66, 1286-1296,

16 Bollard, J. et al. Palbociclib (PD-0332991), a selective CDK4/6 inhibitor, restricts
growth in preclinical models of hepatocellular carcinoma. Gut 66, 1286-1296, doi:10.1136/gutjnl-2016-312268 (2017).

17 Knudsen, E. S., Pruitt, S. C., Hershberger, P. A., Witkiewicz, A. K. \& Goodrich, D. W. Cell Cycle and Beyond: Exploiting New RB1 Controlled Mechanisms for Cancer Therapy. Trends Cancer 5, 308-324, doi:10.1016/j.trecan.2019.03.005 (2019).

18 O'Leary, B. et al. The Genetic Landscape and Clonal Evolution of Breast Cancer Resistance to Palbociclib plus Fulvestrant in the PALOMA-3 Trial. Cancer discovery 8, 1390-1403, doi:10.1158/2159-8290.CD-18-0264 (2018).

19 Haibe-Kains, B. et al. Inconsistency in large pharmacogenomic studies. Nature 504, 389393, doi:10.1038/nature12831 (2013).

20 Garnett, M. J. et al. Systematic identification of genomic markers of drug sensitivity in cancer cells. Nature 483, 570-575, doi:10.1038/nature11005 (2012). doi:10.3892/ijo.2015.2816 (2015). Jeong, Y. et al. Role of KEAP1/NFE2L2 Mutations in the Chemotherapeutic Response of Patients with Non-Small Cell Lung Cancer. Clin Cancer Res, doi:10.1158/1078-0432.CCR19-1237 (2019).

23 Arbour, K. C. et al. Effects of Co-occurring Genomic Alterations on Outcomes in Patients with KRAS-Mutant Non-Small Cell Lung Cancer. Clin Cancer Res 24, 334-340, doi:10.1158/1078-0432.CCR-17-1841 (2018).

24 Jackson, E. L. et al. Analysis of lung tumor initiation and progression using conditional expression of oncogenic K-ras. 15, 3243-3248 (2001).

25 Chiou, S. H. et al. Pancreatic cancer modeling using retrograde viral vector delivery and in vivo CRISPR/Cas9-mediated somatic genome editing. Genes Dev 29, 1576-1585, doi:10.1101/gad.264861.115 (2015).

26 Madisen, L. et al. A robust and high-throughput Cre reporting and characterization system for the whole mouse brain. Nat Neurosci 13, 133-140, doi:10.1038/nn.2467 (2010). eliminate index cross-talk and significantly improve sensitivity of massively parallel sequencing. BMC Genomics 19, 30, doi:10.1186/s12864-017-4428-5 (2018).

28 Pezza, J. A., Kucera, R. \& Sun, L. J. N. E. B. Polymerase fidelity: what is it, and what does it mean for your PCR. (2014).

29 Sinha, R. et al. Index switching causes "spreading-of-signal" among multiplexed samples in Illumina HiSeq 4000 DNA sequencing. 125724 (2017). 
Murray, C. W. et al. An LKB1-SIK Axis Suppresses Lung Tumor Growth and Controls Differentiation. Cancer discovery 9, 1590-1605, doi:10.1158/2159-8290.CD-18-1237 (2019).

31 Cheng, D. T. et al. Memorial Sloan Kettering-Integrated Mutation Profiling of Actionable Cancer Targets (MSK-IMPACT): A Hybridization Capture-Based Next-Generation Sequencing Clinical Assay for Solid Tumor Molecular Oncology. J Mol Diagn 17, 251-264, doi:10.1016/j.jmoldx.2014.12.006 (2015).

\section{Acknowledgements:}

We thank the Laura Andrejka for technical support; A. Orantes for administrative support;

member of the Stanford Animal care staff for excellent animal care; D. Feldser, J. Sage, J.

305 Zhang, and members of the Winslow and Petrov laboratories for helpful comments. C.L. is the

306 Connie and Bob Lurie Fellow of the Damon Runyon Cancer Research Foundation (DRG-2331).

307 W-Y.L. was supported by an American Association of Cancer Research Postdoctoral fellowship

308 (17-40-18-LIN). H.R. was supported by the Druckenmiller Center for Lung Cancer Research at

309 MSK. H.C. was supported by a Tobacco-Related Disease Research Program Postdoctoral

310 Fellowship (28FT-0019). C.D.M was supported by NIH K99-CA226506. M.Y. was supported

311 by a Stanford Dean's fellowship, an American Lung Association senior research training grant,

312 and NIH F32-CA236311. I.P.W was supported by the NSF Graduate Research Fellowship

313 Program and NIH F31-CA210627. This work was supported by NIH R01-CA207133 (to

314 M.M.W and D.A.P.), NIH R01-CA231253 (to M.M.W and D.A.P), NIH R01-CA234349 (to

315 M.M.W and D.A.P.) and Memorial Sloan Kettering Cancer Center Support Grant/Core Grant 316 (P30 CA008748). 


\section{AUTHOR CONTRIBUTIONS}

319 C.L., W.L., M.M.W., D.A.P., and C.M.R. planned the project. C.L. performed bioinformatics

320 and statistical analysis. W.L., H.C., M.M.W., Z.R., and M.Y. performed the mice experiment.

321 H.R. collected the patient response dataset. H.R. and C.L. performed the analysis on human

322 dataset. Z.N.R., I.P.W., and C.D.M. assisted in the mice experiments. C.L., M.M.W and D.A.P.

323 wrote the paper.

324

325

326

\section{COMPETING INTERESTS}

328 D.A.P., I.P.W, and M.M.W are cofounders of D2G Oncology Inc. D.A.P., I.P.W, Z.N.R, C.D.M 329 and M.M.W hold equity in D2G Oncology Inc.

330

331

332

333

334

335

336

337

338

339

340 


\section{Generation of lentiviral vectors}

The design and barcoding of Lentiviral sgRNA/Cre vectors were previously described ${ }^{15}$.

344 Briefly, each vector contains a guide RNA (sgRNA) targeting a specific tumor suppressor gene,

345 a highly diverse barcode region (BC) (total length $23 \mathrm{bp}$, the length of the diverse sequence is

$34615 \mathrm{bp}$ ), and an sgID sequence of $8 \mathrm{bp}$ that is specific to each sgRNA. The BC and sgID regions

347 are adjacent to one another and can be amplified in a single PCR reaction. To eliminate sgID-

348 BC-sgRNA uncoupling driven by lentiviral template switching during reverse transcription of

349 the pseudo-diploid viral genome, we produced each barcoded Lenti-sgRNA/Cre vector

350 separately. We transfected 293T cells with individual barcoded Lenti-sgRNA/Cre plasmids

351 (sgApc, sgAtm, sgAridla, sgCdkn2a, sgKeap1, sgLkb, sgNeo1, sgNeo2, sgNeo3, sgNT1, sgp53,

352 sgRb1, sgRbm10, sgSetd2, and sgSmad4) and packaging plasmids (delta8.2 and VSV-G) using

353 polyethylenimine-based transfection. The supernatant was collected at 48 and 72 hours after

354 transfection. Each lentiviral vector was filtered and concentrated by ultracentrifugation $(25,000 \mathrm{~g}$

355 for 1.5 hours), resuspended in PBS, and frozen in aliquots at $-80^{\circ} \mathrm{C}$. To determine the titer of each

356 vector, we transduced LSL-YFP cells (a gift from Dr. Alejandro Sweet-Cordero/UCSF),

357 determined the percent YFP-positive cells by flow cytometry, and calculated the titer by

358 comparing to a lentiviral preparation of a known titer. Viral vectors were thawed and pooled

359 immediately prior to delivery to mice.

360 Mice, tumor initiation, and drug treatment

361

$$
\operatorname{Kras}^{L S L-G 12 D}(K), R 26^{L S L-T o m a t o}(T), H 11^{L S L-C a s 9} \text { mice (hereafter } K T ; H 11^{L S L-C a s 9} \text { mice) have }
$$

362 been previously described ${ }^{24-26}$. Lung tumors were initiated using a pool of barcoded Lentiviral- 
sgRNA/Cre vectors delivered by intratracheal transduction of mice as previously described ${ }^{15}$.

$3641.1 \times 10^{5}$ and $2.2 \times 10^{4}$ infectious particles in $60 \mu$ of PBS were administered to each $K T$ and

$365 K T ; H 11^{L S L-C a s 9}$ mouse, respectively. Tumor burden was assessed by fluorescence microscopy,

366 lung weight measurement, and histology. Drug treatments were started 15 weeks after tumor

367 initiation.

In the main pharmacogenomic mapping experiment, mice were assigned to eight

369 treatment arms or 8 mice were left untreated for 3 weeks. There were five arms of

370 monotherapies. 6 mice were treated with $100 \mathrm{mg} / \mathrm{kg}$ palbociclib daily by oral gavage; 5 mice

371 were treated with $10 \mathrm{mg} / \mathrm{kg}$ everolimus daily by oral gavage, 6 mice were treated with 100

$372 \mathrm{mg} / \mathrm{kg}$ phenformin daily by oral gavage, 5 mice were treated with $20 \mathrm{mg} / \mathrm{kg}$ paclitaxel every

373 other day by intraperitoneal injection, and 7 mice were treated with $0.3 \mathrm{mg} / \mathrm{kg}$ trametinib daily

374 by oral gavage. For mice treated with drug combinations, the dosing was the same as when each

375 drug was used for monotherapies. 8 mice were treated with the combination of $20 \mathrm{mg} / \mathrm{kg}$

376 paclitaxel every other day by intraperitoneal injection and $0.3 \mathrm{mg} / \mathrm{kg}$ trametinib daily by oral

377 gavage; 7 mice were treated with $50 \mathrm{mg} / \mathrm{kg}$ carboplatin every 5 days by intraperitoneal injection

378 in combination with $20 \mathrm{mg} / \mathrm{kg}$ paclitaxel every other day by intraperitoneal injection; and 6 mice

379 were treated by a combination of three drugs: $50 \mathrm{mg} / \mathrm{kg}$ carboplatin every 5 days by

380 intraperitoneal injection, $20 \mathrm{mg} / \mathrm{kg}$ paclitaxel every other day by intraperitoneal injection, 0.3

$381 \mathrm{mg} / \mathrm{kg}$ trametinib daily by oral gavage.

382 For the palbociclib repeat experiment, 5 mice were left untreated and 5 mice were treated

383 with $100 \mathrm{mg} / \mathrm{kg}$ palbociclib daily by oral gavage. For the negative control experiment, $4 K T$ mice

384 were left untreated, and $5 K T$ mice were treated with a combination of carboplatin, paclitaxel,

385 and trametinib with the same dosing as the main experiment, except that treatment was continued 
for 6 weeks. All mice in all experiments were transduced with the same pool of viral vectors. All experiments were performed in accordance with Stanford University Institutional Animal Care and Use Committee guidelines.

Isolation of genomic DNA from mouse lungs, preparation of sgID-BC libraries, and quantification of tumor size spike-in cell lines divided by the expected number of cells for each spike-in $\left(5 \times 10^{5}\right.$ cells $)$. genomic DNA per mouse. To enable the identification and subsequent computational elimination of possible index-hopped reads in high-throughput sequencing, the sgID-BC region of the

403 integrated Lenti-sgRNA-BC/Cre vectors was PCR amplified from each sample with unique dual

404 indexed primer pairs ${ }^{27}$. Because Illumina short-read sequencing platforms require high library 405 sequence diversity for accurate base calls, we previously added 50\% PhiX phage DNA to each 406 amplicon library before sequencing as recommended by Illumina. To further improve sequencing 407 quality and minimize the use of PhiX DNA, we improved our previous method by adding 6 to 9 408 random nucleotides (Ns) to the flanking ends of both index primers before the sequence-specific 
409 primers. These random bases increase sequence diversity through their high nucleotide diversity

410 and by desynchronizing the barcode regions of the amplicon library (via differing 6-9 nucleotide

411 offsets). This reduced the needed PhiX genomic DNA from 50\% to 10\% and greatly improved

412 Q30 scores.

413 Forward primers were of the form:

414 5'- AATGATACGGCGACCACCGAGATCTACACAGCGCTAG

415 АCACTCTTTCCCTACACGACGCTCTTCCGATCT [N] 6-9.9CGCACGTCTGCCGCGCTG -3’

417 Reverse primers were of the form:

418 5'- CAAGCAGAAGACGGCATACGAGATCGTGAT

419 GTGACTGGACTTCAGACGTGTGCTCTTCCGATCT [N] $]_{6-9}$ CAGGTTCTTGCGAACCTCAT -3’

420 The underlined sequences denote regions that bind the template; the bolded sequences are

421 the dual indexes which are unique to each mouse library pooled on the same sequencing lane; the

422 italicized regions are the Illumina ${ }^{\circledR}$ TruSeq Universal adapter sequence, and the sequences 5' of

423 the Indexes are the P5 and P7 adaptors.

424 We used a single-step PCR amplification of sgID-BC regions. We performed eight 100

$425 \mu$ l PCR reactions per sample (4 $\mu \mathrm{g}$ DNA per reaction) using Q5 High-Fidelity 2x Master Mix

426 (New England Biolabs) ${ }^{28}$ to maximize library sequencing quality. Pooled PCR products were

427 isolated by gel electrophoresis and gel extracted using the Qiagen ${ }^{\circledR}$ MinElute Gel Extraction kit.

428 The concentration of purified PCR products from each sample was determined by Bioanalyzer

429 (Agilent Technologies) and pooled for sequencing. Pooled libraries were cleaned up and size-

430 selected using AMPure XP beads (Beckman Coulter). Libraries were sequenced on an Illumina ${ }^{\circledR}$

431 HiSeq 2500 to generate paired-end 150 bp reads. Unlike patterned flow cells (e.g., HiSeq 4000), 
432 nonpatterned flow cells used by HiSeq 2500 exhibit extremely low index hopping rates ${ }^{29}$. Paired-

433 end sequencing was used to ensure the fidelity of our barcode calls.

\section{Processing reads to identify the sgID and barcode}

435 By systematically improving DNA library preparation and sequencing, we dramatically

436 improved the quality of our barcoded tumor calls. In doing so, our previous DADA2-based

437 denoising approach became obsolete, and a new, more stringent computational analysis pipeline

438 that completely eliminated spurious tumors from PCR and sequencing errors was developed.

439 Read sequences are expected to contain an 8-nucleotide sgID region followed by a 23-

440 nucleotide BC (AANNNNNTTNNNNNAANNNNN). We required both the forward and reverse

441 sequencing reads to match perfectly within the $\mathrm{BC}$ region. This stringent requirement minimizes

442 sequencing errors that will generate sgID-BC reads that do not represent actual clonal tumors

443 within the samples. The FASTQ files of each technical replicate were combined for all analyses

444 except when used for evaluating the reproducibility and quantifying error.

445 FASTQ files were processed to identify the sgID and BC counts for each tumor. The

446 sgID region identified the targeted tumor suppressor gene, which we identified from the forward

447 read alone, again allowing no mismatches or indels relative to the expected sgID sequences. Note

448 that all sgID sequences differ by at least 2 nucleotides from each other, making this step robust to

449 sequencing errors. The number of reads with each unique sgID-BC in each sample were added

450 up to arrive at the size of each putative tumor (in the units of the number of reads). 


\section{Identification of unique tumors from random barcodes and removal of "spurious tumor"}

\section{2 generated by read errors}

PCR and sequencing errors within the random barcode regions may be misinterpreted as

454 unique tumors. These spurious tumors bias the analysis of tumor size distribution, reduce signals

455 of tumor suppression, and potentially confound our ability to detect GSTR using Tuba-seq.

456 Therefore, we used stringent criteria to reduce and even eliminate the effects of PCR and

457 sequencing errors on tumor calls.

The random barcode in the sgID-BC region is 15 nucleotides long. Thus, there is a

459 theoretical potential diversity of $\sim 4^{15}>10^{9}$ barcodes within each lentiviral vector. While the

460 actual diversity in each Lenti-sgRNA/Cre vector is dictated by the number of colonies generated

461 during the plasmid barcoding step, this theoretical diversity guarantees that any two genuine

462 tumors will be very unlikely to have barcodes within a certain Hamming distance from one

463 another. Only approximately one pair of true tumors is expected to have barcodes that are only

464 two nucleotides different from each other when making all pair-wise comparison in a set of 1000

465 tumors. Thus, we expect that most reads within two nucleotides from each other within the same

466 mouse library are most likely due to sequencing/PCR errors. Therefore, we designed a pipeline

467 in which any "tumor" with a barcode that was within a Hamming distance of two from a larger

468 tumor in the same sample was excluded from subsequent analysis. As anticipated, excluded

469 tumors were greatly enriched at the lowest end of the tumor size distribution, and were most

470 often orders of magnitude smaller than the larger tumor with a similar barcode (Mann-Whitney

$471 \mathrm{U}$ test of tumor sizes in the comparison between the removed and the remaining tumors, $\mathrm{P}<10^{-}$

$472{ }^{300}$ ). Thus, we elected to remove such putatively spurious tumors from subsequent analysis.

473 Previously, we combined the smaller "spurious" tumors into the larger tumor with the similar 
474 barcode, as PCR and sequencing errors were more common and constituted a non-negligible

475 fraction of the sequenced library. However, with our improved approach, these spurious tumors

476 constituted only $\sim 0.3 \%$ of all reads and are necessarily more likely to be distinct tumors (as

477 spurious tumors are now rarer), so we did not merge spurious tumors into their larger partners in

478 this analysis. Because of the very small quantity of spurious tumors in our new approach, the

479 decision to merge (or not merge) tumors have negligible effects on tumor size estimates.

480 To determine whether our stringent filtering removed most spurious tumors, we first

481 estimated a False Positive Rate using our spike-in cell lines. We assessed whether any spurious

482 tumors were generated from our spike-in controls for which we know the exact sequence of the

483 sgID-BC. In these spike-ins, any sgID-BC read (i.e., with the spike-in sgID) containing a BC

484 sequence that differs from the three known correct BCs is presumably generated by PCR or

485 sequencing errors. Hence, these anomalous spike-in barcodes can be used to estimate the

486 occurrence of spurious tumors. Before applying our filtering pipeline, we detected many such

487 spurious barcode reads - 843 spurious spike-in "tumors" in a typical sequencing lane (Fig. 1c).

488 Our new stringent filtering removed all of them.

489 We next identified true positive tumors by sequencing the sgID-BC region in each

490 plasmid pool used to generate the lentiviral vectors in the Lenti-sgTS ${ }^{\text {Pool }} /$ Cre pool. Given the

491 tremendous theoretical diversity of our random barcodes, barcodes detected in both the plasmid

492 pool and the mouse lungs are very likely to be genuine. We used these data to assess the

493 presence of spurious tumors in the mouse lung as these should almost never be detected in the

494 plasmid pool. However, a false positive rate cannot be directly measured from this plasmid

495 sequencing data because barcode diversity in the plasmid pool is too high to be exhaustively

496 sequenced to cover every potential barcode, i.e., not all barcodes present in the plasmid pool 
were captured in the barcodes sequenced from the plasmids. Thus, some genuine tumors in the mice will have barcodes not captured in the plasmid sequencing. Nevertheless, in a wellcalibrated analysis pipeline, the rate at which genuine tumors are detected should not vary with tumor size. We determined whether the smaller tumors - which are more likely to be polluted by

501 spurious tumors - exhibit a lower probability of detection in the plasmid pool. Before the

502 application of our filtering procedure, $92.1 \%$ of tumors larger than 1000 cells, but only $87.9 \%$ of

503 tumors smaller than 1000 cells were detected in the plasmid pool (Chi-squared test, $\mathrm{p}<10^{-300}$ ).

504 This decline in the true positive tumor fraction with tumor size suggests that some spurious small

505 tumors exist in our data prior to filtering. However, after our filtering procedure, $92.7 \%$ of

506 tumors larger than 1000 cells and $93.1 \%$ of tumors smaller than 1000 cells were detected in the

507 plasmid pool (Fig. 1d and Supplementary Fig. 1b). This consistency of the true tumor rate

508 across tumor size suggests that filtering effectively eliminated spurious tumors. Along with our

509 analysis of spurious spike-ins, both results suggest that our filtering procedure effectively

510 eliminates spurious tumors.

\section{Summary statistics for characterizing tumor growth}

512 Because GSTR can manifest as a mean effect or a change in the shape of tumor size

513 distributions, we used a variety of summary statistics to characterize tumor growth, including

514 percentiles, Log-normal (LN) mean, geometric mean, and relative tumor burden. Percentiles and

515 LN mean were calculated as previously described ${ }^{15}$. Briefly, percentiles are nonparametric

516 summaries for the distribution. $95^{\text {th }}$ percentile for $\operatorname{sgTS}$ tumors, for instance, is calculated as the

517 size above which we find the top 5\% of the largest $s g T S$ tumors and then dividing it by the

518 corresponding number for the tumors with inert sgRNAs (Inert tumors). The resulting number is

519 dimensionless. The LN mean is the maximum likelihood estimator of the mean number of 
520 neoplastic cells given a log-normal distribution of tumor sizes. The LN mean of sgTS tumors is

521 relative to the $\mathrm{LN}$ mean of the Inert tumors. The geometric mean is defined as the product of all

522 tumor sizes raised to the inverse of the number of tumors. For calculation, we compute the

523 arithmetic mean of the logarithm-transformed values of tumor sizes and then use the

524 exponentiation to return the computation to the original scale. The geometric mean is

525 proportional to the average growth rate of tumors. Relative tumor burden is calculated as

527 Developing unbiased procedures for the detection of genotype-specific drug effects

530 variation among mice such as (1) variation in the efficiency of viral delivery and the resulting

531 differences in tumor number and total tumor burden across mice and (2) variation in the library

532 sequencing depth (each sgID within a mouse is part of the same DNA library reaction).

533 However, in the current analysis of genotype-specific drug response, we needed to compare

534 tumor sizes between the two groups of mice - the untreated and the treated - rather than only

535 comparing the relative behaviors of tumors of different genotypes within mice and then

536 aggregating the signals across mice. Thankfully, our multiplexed pool of tumor genotypes still

537 allows us to account for variations among mice, as these effects are still common to all imparted

538 genotypes. First, however, we must generate a genotype-nonspecific null model of response.

539 Note that because we used the same viral pool to initiate tumors in all treated and untreated mice,

540 the initial relative representation of transduced epithelial cells containing each Lenti-sgRNA/Cre

541 is constant and does not vary across mice. Nevertheless, our null model does not assume that the 
542 total numbers of initiated tumors among mice are invariant; only the proportions of initiated

543 tumors with different sgRNAs (sgIDs) remain constant.

\section{Null model of tumor responses with no genotype-specificity}

545 We assume that the therapy affects all tumors proportionally to their sizes (proportional

546 size reduction) such that the size of each tumor changes from $\mathrm{X}$ to $\mathrm{X}_{1}=\mathrm{X} \times S$ after the drug

547 treatment, where $S$ is the proportion of remaining cancer cells. In other words, we assume that

548 the therapy kills individual cancer cells with a probability $1-S$ that is independent of the tumor

549 size. Under the null model $\left(\mathrm{H}_{0}\right)$ of no genotype-specific drug responses, $S$ is constant and does

550 not depend on the genotype of the tumor. Under the alternative model $\mathrm{H}_{1}, S$ varies depending on

551 the genotype: $S_{\mathrm{sgID}, \mathrm{j}}=S_{\mathrm{Inert}} \times\left(1+G_{\mathrm{j}}\right)$, with $G_{j}$ representing the Genotype Specific Therapeutic

552 Response (GSTR) of tumors generated by viruses with the specified sgID to the $\operatorname{drug} \mathrm{j}$. If $G_{\mathrm{j}}>0$,

553 the inactivation of the tumor suppressor associated with that sgID confers relative resistance; if

$554 G_{\mathrm{j}}<0$, the inactivation of the tumor suppressor associated with that sgID confers relative

555 sensitivity.

\section{Selection of a size cutoff in untreated mice}

557 To consider how tumor size distributions shift between the null and alternative models,

558 we must first choose the range of the tumor size distribution to consider. In general, we want to

559 consider tumors that are large enough to be consistently detected regardless of the sequencing

560 depth and PCR efficiency in both treated and untreated mice, while using as many tumors as

561 possible to maximize the statistical power. As shown below, the most extreme treatment reduced

562 tumor sizes by $\sim 87 \%$ ( $S=0.13$, Supplementary Fig. 6d). While the depth of sequencing varies

563 across mice and treatments, we want to reliably identify tumors in each treated and untreated 
mouse. The most effective treatment group has the smallest tumors after treatment, for which

565 the depth of sequencing was $\sim 10$ cells/read. Thus, we chose to use the cutoff of $L=1000$ cells in

566 the untreated mice, as tumors will not shrink below 100 cells or 10 reads in all treated mice,

567 allowing reliable detection and accurate size estimates of tumors in each mouse. Supplementary

568 Fig. 8 shows that our results are robust to shifting the cutoff to 500 or 1500 cells.

\section{Calculation of proportional size-reduction as the drug effect}

We first find the value of the tumor reduction factor $S$ that leads to the best match

571 between the distributions of Inert tumors between the treated and untreated group under our

572 model of proportional tumor reduction. We take the following steps to calculate $S$. For each

573 possible value of $S$ between 0 (tumors are completely eliminated) and 2 (tumors doubled in size

574 after treatment), we reduce the sizes of each tumor in the untreated group by $S$ and calculate the

575 number of such "shrunk" tumors whose sizes remain above or equal to 1000 cells. We then find

576 the value of $S$ such that the median number of such shrunk tumors across all the untreated mice is

577 closest to the median of the number of observed tumors with the size above or equal to 1000

578 cells across all the mice in the treated group. Specifically, we use the binary search algorithm to

579 determine the $S$ that minimizes the difference between the median numbers of tumors of the

580 treated and untreated groups. We choose to find the $S$ that matches the median number of tumors,

581 rather than the mean across the two groups, as the median is not affected by the outlier mice with

582 very low or very high numbers of tumors. Since we have prior knowledge that drugs will not

583 increase overall tumor size, an estimated $S$ larger than 1 is probably due to mouse-to-mouse

584 variations. Thus we set $S$ to 1 when it was estimated to be larger than 1 . In Supplementary Fig.

5859 , we showed that our estimation is robust to the inaccurate estimation of $S$, and the power will

586 only be reduced slightly when $S$ was not accurately estimated. 


\section{Approach 1: Relative tumor number (ScoreRTN)}

Our first approach defines response as the number of tumors that exceed a minimum size

590 threshold. The intuition is that, given a known tumor reduction factor $S$ of the drug, the null

591 hypothesis for each genotype is that the number of tumors above the cutoff $L$ in the untreated

592 mice should match the number above the new cutoff $L \times S$ in the treated mice. If a GSTR exists

593 (the alternative model), then the tumors with a specific sgID (i.e., tumors with a particular tumor

594 suppressor inactivated) are more resistant to the drug than the Inert tumors and more of such

595 tumors should remain above the adjusted cutoff of $L \times S$ than expected, while if they are more

596 sensitive, then fewer of such tumors should remain above the adjusted cutoff of $L \times S-$ rejecting

597 the null hypothesis in either case.

To test this null hypothesis, we first calculate the ratio of the number of tumors above the

599 cutoff $L$ in the untreated mice of a particular $\operatorname{sgID}$ to that of the Inert tumors $\left(R T N_{\mathrm{i}, \mathrm{j}, \mathrm{L}}\right)$,

600

601

for all mice $k$ and all tumors equal or larger than $L$

where $C_{i, j, k}$ is the total number of tumors observed in mouse $k$ in treatment group $j(j=$ untreated

604 here) carrying sgID $i$ above the cutoff $L$. We then calculate the similar ratio for the treated mice

605 with a modified cutoff $L \times S$,

606

$$
R T N_{i, j, L \times S}=\frac{\sum_{k} \mathrm{C}_{i, \mathrm{j}, \mathrm{k}}}{\sum_{k} \mathrm{C}_{\text {Inert }, \mathrm{j}, \mathrm{k}}} \text { for all mice } \mathrm{k} \text { and all tumors larger than } L \times S
$$

607 The null hypothesis can then be expressed as the expectation that 


$$
R T N_{i, \text { untreated }, L}=R T N_{i, j, L \times S}
$$

or alternatively that:

$$
\operatorname{ScoreRTN}_{i, j}=\log _{2}\left(\frac{R T N_{i, j, L \times S}}{R T N_{i, \text { Untreated }, L}}\right)=0
$$

611 Under the alternative hypothesis where $\operatorname{ScoreRTN} N_{i, j} \neq 0$, a positive sign of $\operatorname{ScoreRTN} N_{i, j}$

612 suggests that the tumors with a particular sgID are more resistant than the Inert tumors, while a

613 negative sign suggests the tumors are more sensitive than Inert tumors.

614 Although directly comparing the size or number of tumors above a constant cutoff

615 (e.g.,1000 cells), for both the untreated and treated groups may seem intuitive and simpler, such

616 a comparison generates complex expectations of tumor number that depend both on the

617 distributions of tumor sizes prior to treatment and the magnitude of the drug effect

618 (Supplementary Fig. 3).

\section{Approach 2: Relative geometric mean (ScoreRGM)}

621 The second metric, Score $R G M$, compares the geometric mean of tumors carrying sgID $i$

622 relative to the Inert tumors in the untreated and treated groups. The intuition is that if we analyze

623 a comparable number of tumors in the untreated and treated mice when there is no GSTR, the

624 relative growth advantage of tumors carrying a specific $\operatorname{sgID}(\operatorname{sgID} i$ ) relative to Inert tumors,

625 represented by the relative geometric mean, will remain constant. Under the alternative model, if

626 the tumors with a specific $\operatorname{sgID}(\operatorname{sgID} i)$ are more resistant to the drug than the Inert tumors, then

627 the relative geometric mean for $\operatorname{sgID} i$ will be larger in the treated group, while if they are more

628 sensitive, then the relative geometric mean for $\operatorname{sgID} i$ will be smaller. While $R T N$ does not use

629 the numeric value of tumor size other than comparing it with the cutoff, (i.e., a tumor with size 
6301001 cells and a tumor with size $10^{7}$ cells are both counted as a single tumor above the cutoff of

6311000 cells), $R G M$ incorporates such tumor size profile information. Hence, $R G M$ and $R T N$ are

632 not entirely redundant as they incorporate different information about GSTR. Based on power

633 analysis, ScoreRTN is a more sensitive metric in detecting GSTRs (Fig $\mathbf{2 b}$, c, and

634 Supplementary Fig. 4), particularly when only smaller tumors show GSTR (Supplementary

635 Fig. 5a). However, when only larger tumors show GSTR, the ScoreRGM is more likely to

636 capture it, as large tumors are unlikely to fall below the given size threshold. For instance, in an

637 extreme case, when only large tumors with over 4000 cells show resistance, ScoreRTN will fail

638 to capture the signals that ScoreRGM identifies with reasonable power (Supplementary Fig.

$6395 \mathbf{b})$.

640 We denote the total tumor count $(T)$ with a certain sgRNA $(i)$ in an individual mouse $(k)$

641 in the treated group $(j)$ as $T_{\mathrm{i}, \mathrm{j}, \mathrm{k}}$. Here, we do not limit tumors to those above 1000 cells but rather

642 count any tumor with greater than or equal to 2 reads (after the stringent filtering described

643 above) as a tumor. For an untreated mouse, the proportion of initiated tumors of each sgID can

644 be approximated by $R_{\mathrm{i}}$, the ratio of $T_{i, \text { untreated, } k}$ to $T_{\text {Inert, untreated, } k}$ :

645

$$
R_{i}=\operatorname{median}\left(\frac{T_{i, \text { untreated }, k}}{T_{\text {Inert }, \text { untreated }, k}} \mid \text { for all mice } k\right)
$$

We then take the top $N$ tumors with $\operatorname{sgRNA} i$ from mouse $k$ treated by $\operatorname{drug} j$ as:

$$
\mathrm{N}_{i, j, k}=\mathrm{C}_{i, j, k} \times \mathrm{R}_{i}
$$

648 where $C_{i, j, k}$ is the total number of Inert tumors observed in each mouse above the cutoff $L \times S$

649 ( $S=1$ for the untreated group), and then we calculate the geometric mean for all tumors

650 containing the sgID and Inert tumors across all mice in the group.

651 The score for the relative geometric mean is calculated as: 


$$
\operatorname{ScoreRGM} M_{i, j}=\log _{2}\left(\frac{\mathrm{GM}_{\mathrm{i}, \mathrm{j}} / \mathrm{GM}_{\text {Inert,j }}}{\mathrm{GM}_{\mathrm{i}, \text { untreated }} / \mathrm{GM}_{\text {Inert,untreated }}}\right)
$$

653 where $\mathrm{GM}_{\mathrm{i}, \mathrm{j}}$ is the geometric mean for tumors containing $\operatorname{sgID} i$ in treatment group $j$ in the

654 selected $N$ tumors. Under the null hypothesis, $\operatorname{Score} R G M_{i, j}=0$. Under the alternative

655 hypothesis where $S \operatorname{coreR} G M_{i, j} \neq 0$, a positive sign of $\operatorname{Score} R G M_{i, j}$ suggests that the tumors

656 with a particular sgID are more resistant than the Inert tumors, while a negative sign of the score

657 suggests that these tumors are more sensitive than the Inert tumors.

\section{Evaluating whether ScoreRTN and ScoreRGM are biased}

660 untreated mice to subjected to a simulated treatment with no GSTR (specifically, the 5 untreated

661 mice from the palbociclib repeat experiment). This represents an ideal scenario where we know

662 exactly how each tumor responded to the treatment (because we generate the responses through

663 simulations), and we are able to measure the exact tumors with and without treatment.

664 Specifically, as shown in Supplementary Fig. 3a, we reduce the tumor sizes of all tumors in the

665 five untreated mice by 50\% as the "treated" tumors (Supplementary Fig. 3b). For a biased

666 method illustrated in Supplementary Fig. 3c, e, f, which fails to consider the size reduction of

667 tumors due to drug treatment by using the constant 1000 cell cutoff in both untreated and treated

668 mice, the relative tumor number and relative geometric mean does not remain constant between

669 treated and untreated mice (Supplementary Fig. 3e, f). This is because a constant cell number

670 cutoff effectively compares different proportions of the distributions of the inert and TS-

671 inactivated tumors in the treated and untreated mice. 
On the other hand, using the adaptive cutoff method introduced above, no false signals of

673 ScoreRTN and ScoreRGM were observed (Supplementary Fig. 3d, g, h), because we are

674 comparing the matched portions of the distributions for the Inert tumors and tumors of each

675 genotype (tumor with each sgID) between untreated and treated mice. Therefore, these two

676 statistics appear unbiased.

\section{Bootstrapping the tumors}

When generating null distributions of scores and calculating the p-values, we performed

679 bootstrap resampling on tumors. During bootstrapping, we consider tumor size variations both

680 across mice and within a mouse using a nested resampling approach: first, we bootstrapped mice

681 in the untreated and/or the treated group to generate pseudogroups of mice, and then within each

682 mouse, we bootstrapped all observed tumors carrying each sgID.

\section{Generate a null distribution of ScoreRGM and ScoreRTN}

To generate a null distribution of ScoreRTN and ScoreRGM in the absence of GSTR, we sampled with replacement the same number of mice as in the treated group from the 8 untreated

686 mice and applied estimated drug effects $S$ on the tumors from the "treated" groups. For each

687 bootstrap run, we re-estimated $S$ and calculated the values of ScoreRTN and ScoreRGM. 
To see how the distribution of observed ScoreRTN and ScoreRGM of $\operatorname{sgID} i$ deviates

695 from the null distribution, we compared the distribution of the two observed scores to that

696 calculated from (1) simulated data with no GSTR for all sgIDs and to (2) simulated data with no

697 GSTR for sgID $i$ to determine the $P$-value. For each comparison, we sample values from both

698 distributions and calculate the $P$-value as the fraction of times when their differences are not in

699 the same direction as the observed score, i.e., how often do we see equal or more extreme scores

700 under the null distribution. To be conservative, the maximum of the two $P$-values calculated

701 from the two comparisons were reported as the $P$-value. This bootstrap process is performed $10^{8}$

702 times to calculate the $P$-values.

\section{Power analysis for ScoreRTN and ScoreRGM in our study}

704

To estimate the sensitivity (True Positive Rate) and specificity (1-False Positive Rate) of

705

our study (Fig. 2b, c), we sampled with replacement eight and five mice (minimum number of

706

treated mice in the pharmacogenetics mapping experiment) from the eight untreated mice, as the

707 "untreated" and "treated" groups, respectively. We then reduce all tumor sizes to $50 \%$ as the

708 drug effect $(S=0.5)$ and apply an input $G=-50 \%,-20 \%, 0 \%$ (no genotype-specific drug

709 response), 10\%, 20\%, and 50\% to each non-Inert sgIDs by additionally changing each tumor

710 sizes by the corresponding proportions. For instance, $G=20 \%$ means the overall drug effect on

711 tumors carrying the $\mathrm{ggRNA}$ is $(1-50 \%) \times(1+20 \%)=60 \%$. Sensitivity is calculated as the

712 probability of detecting preassigned true genotype-specific interactions, and specificity is

713 calculated as the proportion of sgIDs correctly identified as having no GSTR when $G=0$. A total

714 of 100 runs of simulations of the 11 tumor suppressor gene targeting sgIDs were performed for 
715 each preassigned $G$. We adjust the cutoff for $P$-values and calculated a series of sensitivity and

716 specificity values to plot the receiver operating characteristic (ROC) curve.

\section{Power analysis for ScoreRTN and ScoreRGM for various sample sizes}

719 we sampled the same number of mice $(5,10$, or 20 mice) with replacement from the 8 untreated

720 mice as the untreated and treated group. Similar to the previous section of Power analysis using 8

721 and 5 mice respectively for untreated and treated mice, we apply $G$ of various magnitudes and

722 plotted the ROC curve when we use 5, 10, and 20 mice for each group in our experiment,

723 respectively.

\section{Power analysis for ScoreRTN and ScoreRGM for tumor size-dependent GSTR}

725 We evaluate the effectiveness of ScoreRTN and ScoreRGM in capturing GSTR when the

726 input $G$ is not a constant factor constant across tumors with various sizes (Supplementary Fig.

727 5). We apply a truncated effect of $G$ where 1) only tumors smaller than 4000 cells showed

728 genotype-specific sensitivity or 2) only tumors larger than 4000 cells showed genotype-specific

729 resistance. The rest of the tumors are simulated to respond the same as Inert tumors to the drug.

730 We calculated the ROC curves for the two statistics using 5 mice in both the untreated and

731 treated groups.

732 Apart from ScoreRTN and ScoreRGM, which are based on the relative tumor number and

733 relative geometric mean, other summary statistics, such as relative $\mathbf{L N}$ mean (ScoreRLM), can

734 also be used to identify GSTR. The score for the relative LN mean is calculated as:

735

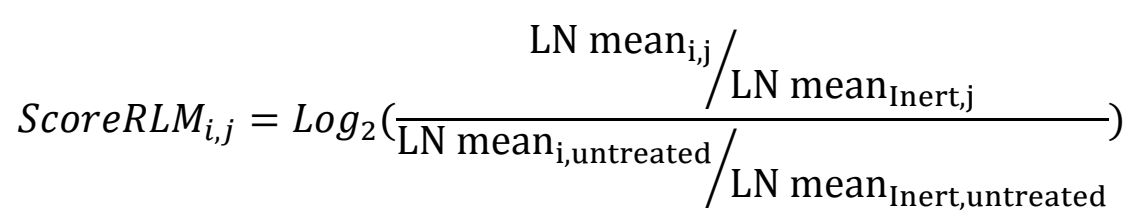


736 where $\mathrm{LN}$ mean $_{\mathrm{i}, \mathrm{j}}$ is the $\mathrm{LN}$ mean for tumors containing $\operatorname{sgID} i$ in treatment group $j$ in the

737 selected $N$ tumors. We compared and contrasted the performance of the three metrics when the

738 GSTR is not a constant factor, and show that if we have a good reason to believe that only very

739 large tumors show genotype-specific responses, ScoreRLM is the best metric among the three.

740 Otherwise, the other two metrics, ScoreRTN and ScoreRGM, will outperform ScoreRLM.

\section{Evaluating whether inaccuracy of drug effect $(S)$ estimation influences estimates of GSTR}

We also evaluate the impact of inaccurately estimating drug effects on our estimates of

743 GSTR (Supplementary Fig. 9). Instead of estimating the effect size as described in section

744 "calculation of proportional size-reduction as drug effect", we assign $S$ to be a constant value,

745 taking three discrete values $0.3,0.5$, and 0.7 when we know the simulated truth of drug effect is

$746 S=0.5$. For each simulated scenario, we calculated the specificity and sensitivity and plotted the

747 ROC curve for detecting $G=+20 \%$ using 5 mice in both the untreated and treated groups

\section{8 (Supplementary Fig 9).}

\section{Calculating ScoreGSTR $(\widehat{G})$ as the combined score}

751 on tumor size distribution, it would be helpful to have a single combined score. We calculated a

752 combined score of GSTR $(\widehat{G})$ by taking the inverse variance weighted average of ScoreRTN and

753 ScoreRGM, then converting it to the linear scale (Fig 2f, Supplementary Fig 4c).

754

$$
\text { ScoreGSTR }=\left(\frac{\text { ScoreRTN }}{\sigma_{\text {ScoreRTN }}^{2}}+\frac{\text { ScoreRGM }}{\sigma_{\text {ScoreRGM }}^{2}}\right) /\left(\frac{1}{\sigma_{\text {ScoreRTN }}^{2}}+\frac{1}{\sigma_{\text {ScoreRGM }}^{2}}\right)
$$

$$
\widehat{G}=2^{\text {ScoreGSTR }}-1
$$

756 If $\widehat{G}>0$, GSTR is resistant, and if $\widehat{G}<0$, GSTR is sensitive. 
To be very conservative, for the combined score to be called significant, we require at

758 least one significant $P$-value $(P<0.05)$, and one marginally significant $P$-value $(P<0.1)$ for the

759 two statistics ScoreRTN and ScoreRGM.

\section{Evaluate the consistency for choosing various cell cutoffs and control sgIDs}

761 We use a cutoff of 1000 cells for most parts of the analysis, but we also wanted to

762 evaluate whether our results are robust to using higher and lower cell number cutoffs. Therefore,

763 we adjusted the cutoff to 500 cells and 1500 cells and re-identified the significant GSTR under

764 each scenario (Supplementary Fig. 8a).

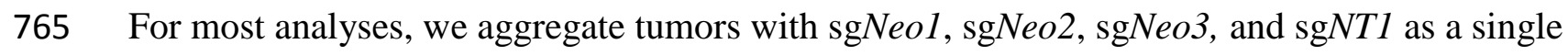

766 Inert control sgRNA to determine the baseline. We also explored whether excluding any of the

767 four sgIDs associated with Inert sgRNAs from the control group would yield similar results. For

768 comparison, we calculated the Spearman correlation and linear correlation, identified significant

769 cases of GSTR, and the overall direction of GSTR for each scenario compared with using all

770 four sgIDs associated with Inert sgRNA.

\section{Comparing with human cell line response data}

772 The drug sensitivity data from human cell lines were downloaded from the Genomics of

773 Drug Sensitivity in Cancer (GDSC) database (www.cancerrxgene.org) ${ }^{3}$. GDSC used logic-based

774 modeling to quantified how genetic alterations in 1001 human cancer cell lines are correlated

775 with sensitivity to various drugs. There is a limited number of LUAD cell lines in the database.

776 Therefore, we focused on comparing the results from Pan-cancer cell lines. All 5 monotherapies

777 used in our study were assessed by GDSC. Except for Keap1 and Rbm10, which are not reported 
778 for everolimus and paclitaxel, the GSTR of all other 51 gene-drug pairs were quantified by

779 GDSC. The effect size and FDR-corrected $P$-values were used for comparison.

780 Hierarchical clustering of GSTR for treatment and genes

781 To better visualize the similarity of the GSTR profiles, we performed hierarchical

782 clustering with complete linkage on $\widehat{G}$ to visualize the relationship across different genes and

783 across different therapies (Supplementary Fig 7c, d). Therapies or genes that are similar to each

784 other in genotype-specific responses are clustered together.

785

786

787

788

789

790

791

792

793

794

795

796

797 occurring mutations. 


\section{FIGURE LEGENDS}

801 Fig. 1. Optimization of tumor-barcoding coupled with high-throughput barcode sequencing 802 (Tuba-seq) for the analysis of genotype-specific therapy responses (GSTRs) in vivo.

803 a. Overview of Tuba-seq pipeline to uncover GSTRs. The Lenti-TS ${ }^{\text {Pool} / C r e ~ v i r a l ~ p o o l ~ c o n t a i n s ~}$

804 barcoded vectors with sgRNAs targeting 11 putative tumor suppressors that are frequently

805 mutated in human lung adenocarcinoma. Tumors are initiated in either $\mathrm{Kras}^{L S L-G 12 D /+} ; R^{2} 6^{L S L-T o m}$

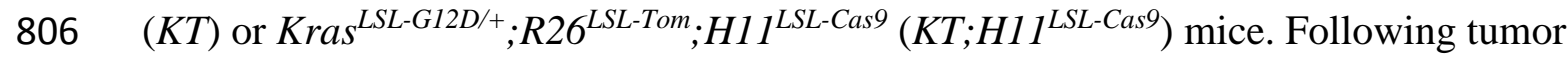

807 development, mice are treated with therapies, and barcode sequencing libraries are prepared from 808 each tumor-bearing lung.

809 b. Comparison of our current pipeline with our previous Tuba-seq pipeline (Rogers et al. 2017).

810 c. Stringent filtering effectively eliminated spurious tumors. Analysis of the barcodes associated 811 with the sgID specific for the Spike-in control cells (3 cell lines with a defined sgID-BC added at

$8125 \times 10^{5}$ cell/sample as the benchmark) enables identification of recurrent barcode reads generated

813 from sequencing and other errors (Spurious tumors). Data is from a typical lane of 22

814 multiplexed Tuba-seq libraries from $K T ; H 11^{L S L-C a s 9}$ mice with Lenti-TS ${ }^{\text {Pool} / C r e ~ i n i t i a t e d ~ t u m o r s . ~}$

815 d. The percent of tumors with barcodes validated within the lentiviral plasmid pool is constant

816 across tumor sizes. As sequencing and other processing errors are most likely to create small

817 spurious tumors, this finding suggests that tumors detected by Tuba-seq represent real clonal 818 expansions of barcoded cells.

819 e. The relative size of tumors of each genotype in $K T ; H 11^{L S L-C a s 9}$ mice 18 weeks after tumor

820 initiation with Lenti-sgTS ${ }^{\text {Pool}} /$ Cre. The relative sizes of tumors at the indicated percentiles were

821 calculated from the tumor size distribution of all tumors in 5 mice. Error bars show $95 \%$

822 confidence intervals.

824 Fig. 2. Tuba-seq quantifies genotype-specific therapeutic responses (GSTR) for multiple 825 therapies.

826 a. Data analysis pipeline to identify GSTR by comparing the relative tumor number (ScoreRTN)

827 and relative geometric mean (ScoreRGM) between tumors containing a tumor suppressor

828 targeting sgRNA and Inert tumors in the untreated and treated mice. 
829 b. The sensitivity and specificity of ScoreRTN estimated from simulations of preassigned drug 830 effect $(S=0.5)$ and GSTR (various $G$ ). There is no genotype-specific response when $G=0 . G$ of $83120 \%$ means the tumors with the sgRNA were reduced by an additional $20 \%$ in size.

832 c. The sensitivity and specificity of ScoreRGM estimated from the same simulation as in b.

833 d. Likely mechanism of action for monotherapies used for treatments.

834 e. Timeline of the experiment. Tumors were initiated in KT;H1 $1^{L S L-C a s 9}$ mice with the barcoded 835 Lenti-sgTS $S^{\text {Pool}} / \mathrm{Cre}$. Three weeks of treatment was initiated after 15 weeks of tumor growth.

836 f-h. $\hat{G}$ calculated from the inverse variance weighted average of ScoreRTN and ScoreRGM for 837 the pharmacogenomic mapping experiment (f), negative control experiment in $K T$ mice (g) and 838 palbociclib repeat experiment $(\mathbf{h})$. Stars represent significant cases.

839 i. Comparison of our identified GSTRs with those from the Genomics of Drug Sensitivity in 840 Cancer (GDSC) database. Stars represent significant cases.

841 j. Kaplan-Meier curve (with 95\% confidence interval in shading) of time-to-next-treatment 842 (months) for patients with or without KEAP1 mutations with metastatic oncogenic KRAS-driven 843 lung adenocarcinoma to platinum-containing chemotherapy. The number of patients in each 844 group is shown. $P$-values were calculated from the Mantel-Haenszel test.

845 k. Responses of patients with metastatic oncogenic $K R A S$-driven lung adenocarcinoma to 846 platinum-containing chemotherapy are consistent with KEAP1 inactivation leading to resistance.

847 KEAP1 mutations are significantly correlated with a higher hazard ratio for time-to-next848 treatment. 
bioRxiv preprint doi: https://doi.org/10.1101/2020.01.28.923912; this version posted January 29, 2020. The copyright holder for this preprint (which was not certified by peer review) is the author/funder. All rights reserved. No reuse allowed without permission.

Li and Lin et al.

a

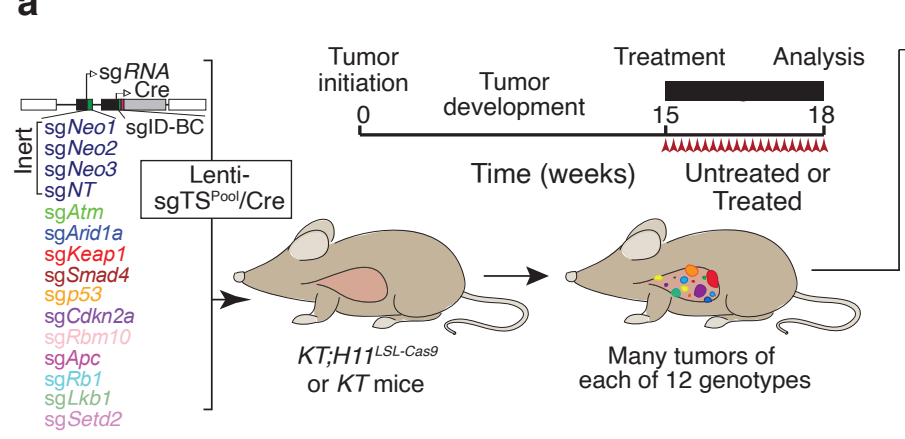

b

\begin{tabular}{lll} 
& Rogers et al. & This manuscript \\
\hline $\begin{array}{l}\text { Viral } \\
\text { production }\end{array}$ & Pooled & $\begin{array}{l}\text { Each vector prepared } \\
\text { seperately } \\
\text { (eliminate template switching) }\end{array}$ \\
$\begin{array}{l}\text { Library } \\
\text { preparation }\end{array}$ & Taq polymerase & $\begin{array}{l}\text { Q5 polymerase } \\
\text { (reduce PCR errors) } \\
\text { Dual unique indexing } \\
\text { (eliminate index hopping } \\
\text { during sequencing) }\end{array}$ \\
Single indexing & $\begin{array}{l}\text { Paired end } \\
\text { (reduce "tumors" created } \\
\text { by sequencing errors) } \\
\text { Sequencing }\end{array}$ & Single end \\
$\begin{array}{l}\text { Read } \\
\text { processing } \\
\text { and tumor } \\
\text { calling }\end{array}$ & $\begin{array}{l}\text { DADA2 } \\
\text { clustering } \\
\text { No restriction } \\
\text { on BC length }\end{array}$ & $\begin{array}{l}\text { Remove spurious tumors } \\
\text { (eliminate "tumors" created } \\
\text { by PCR and sequencing errors) }\end{array}$ \\
\hline
\end{tabular}

C

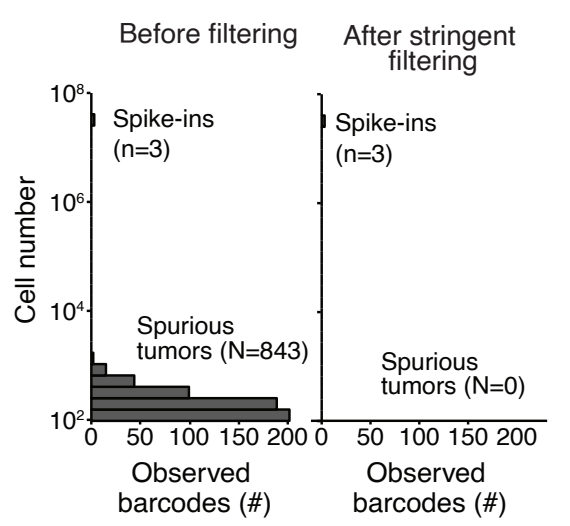

d

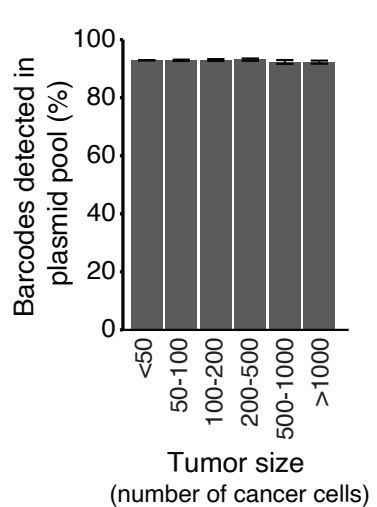

e

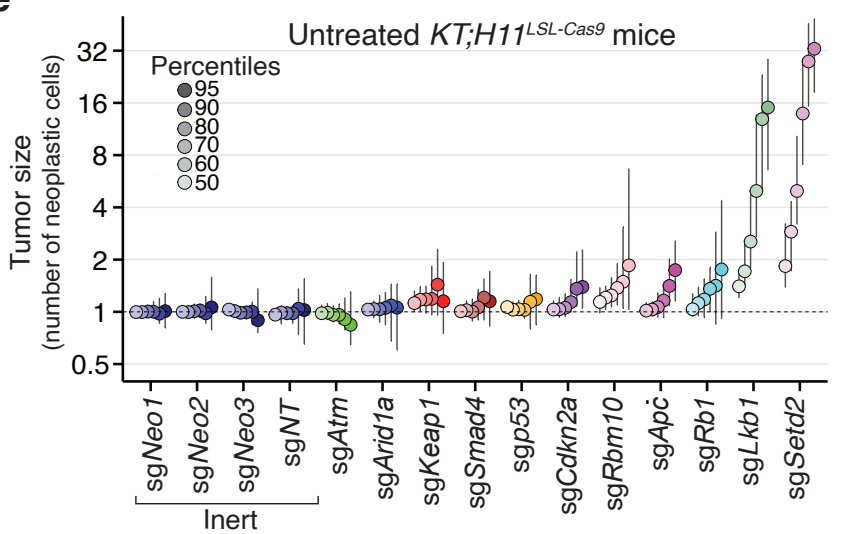

Figure 1. Optimization of tumor-barcoding coupled with high-throughput barcode sequencing (Tuba-seq) for the analysis of genotype-specific therapy responses (GSTRs) in vivo.

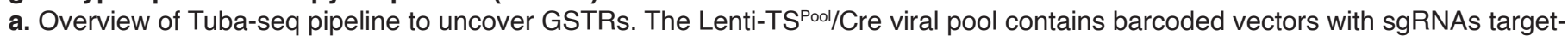
ing 11 putative tumor suppressors that are frequently mutated in human lung adenocarcinoma. Tumors are initiated in either $K_{r^{2} s^{L L-G 12 D /+} ; R 26^{L S L-T o m}(K T)}$ or Kras ${ }^{L L L-G 12 D /+} ; R 26^{L S L-T o m} ; H 11^{L S L-C a s 9}\left(K T ; H 11^{L S L-C a s 9}\right)$ mice. Following tumor development, mice are treated with therapies, and barcode sequencing libraries are prepared from each tumor-bearing lung.

b. Comparison of our current pipeline with our previous Tuba-seq pipeline (Rogers et al. 2017).

c. Stringent filtering effectively eliminated spurious tumors. Analysis of the barcodes associated with the sgID specific for the Spike-in control cells ( 3 cell lines with a defined sgID-BC added at $5 \times 10^{5}$ cell/sample as the benchmark) enables identification of recurrent barcode reads generated from sequencing and other errors (Spurious tumors). Data is from a typical lane of 22 multiplexed

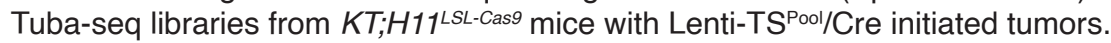

d. The percent of tumors with barcodes validated within the lentiviral plasmid pool is constant across tumor sizes. As sequencing and other processing errors are most likely to create small spurious tumors, this finding suggests that tumors detected by Tuba-seq represent real clonal expansions of barcoded cells.

e. The relative size of tumors of each genotype in $K T ; H 11^{L S L-C a s 9}$ mice 18 weeks after tumor initiation with Lenti-sgTSPool $\mathrm{Cre}^{\mathrm{L} \text {. The }}$ relative sizes of tumors at the indicated percentiles were calculated from the tumor size distribution of all tumors in 5 mice. Error bars show $95 \%$ confidence intervals. 
bioRxiv preprint doi: https://doi.org/10.1101/2020.01.28.923912; this version posted January 29, 2020. The copyright holder for this preprint (which was not certified by peer review) is the author/funder. All rights reserved. No reuse allowed without permission.

Li, Lin et al.

a

Quantify tumor size distributions in untreated and treated mice

Estimate and correct for drug effects on Inert tumors

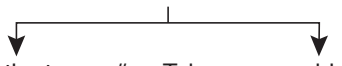

Calculate the tumor \# Take comparable \# of tumors relative to Inert tumors for each sgID from untreated for each sgID and treated group of mice $\downarrow$
$\begin{gathered}\text { Estimate GSTR by } \\ \text { determining the }\end{gathered}$
Estimate GSTR by
determining the determining the
Relative Tumor Number
Relative Geometric Mean ScoreRTN-ScoreRGM

Weighted average of metrics as the best estimator for GSTR $(\hat{\boldsymbol{G}})$

d

\begin{tabular}{lr} 
Drug & Mechanism of Action \\
\hline Palbociclib & Cdk4/6 inhibition \\
Everolimus & mTOR inhibition \\
Phenformin & Mitochondrial dysfunction \\
Paclitaxel & Microtubule stabilization \\
Trametinib & Mek1/2 inhibition \\
Carboplatin & DNA adducts \\
\hline
\end{tabular}

e Tumor initiation

는

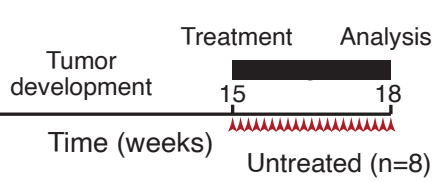

Palbociclib (Palbo; $n=6$ )

Everolimus (Evero; $\mathrm{n}=5$ )

Phenformin (Phen;n=6)

Paclitaxel (Ptx;n=5)

Trametinib (Tra;n=7)

Paclitaxel+Trametinib (Ptx+Tra;n=8)

Carboplatin+Paclitaxel $(\mathrm{C}+\mathrm{Ptx} ; \mathrm{n}=7)$

Carboplatin+Paclitaxel+Trametinib $(\mathrm{C}+\mathrm{Ptx}+\mathrm{Tra} ; \mathrm{n}=6)$ b

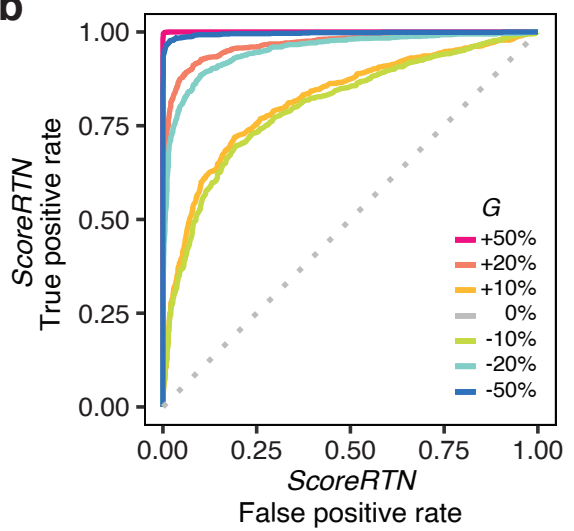

f

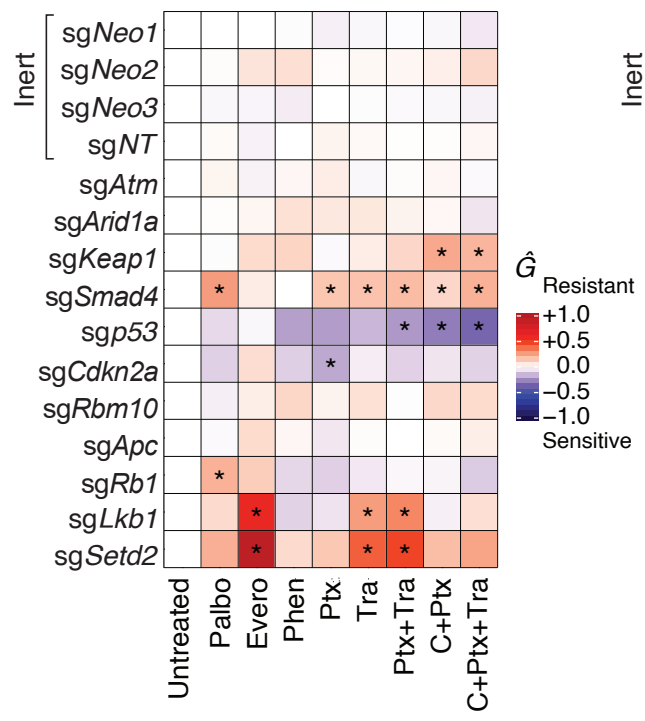

g

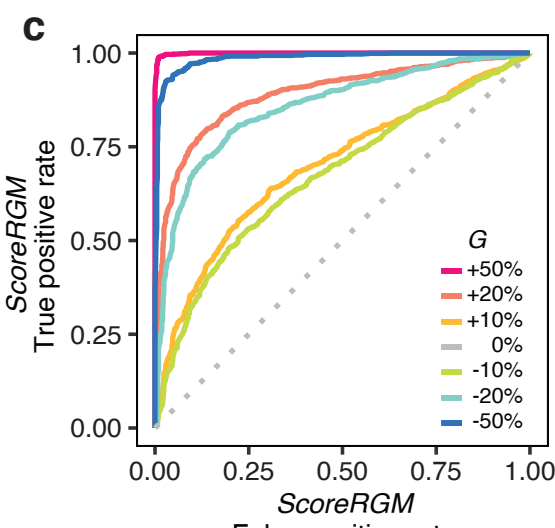

False positive rate

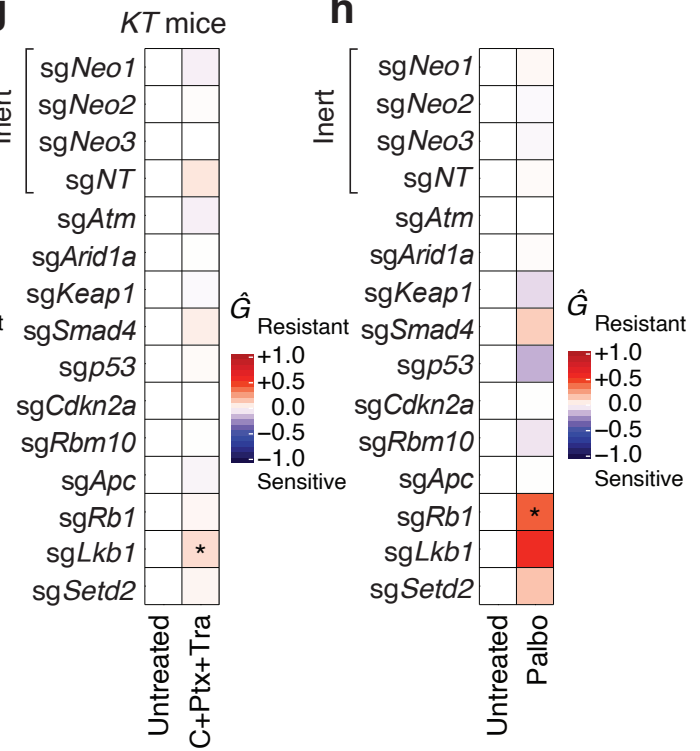

i

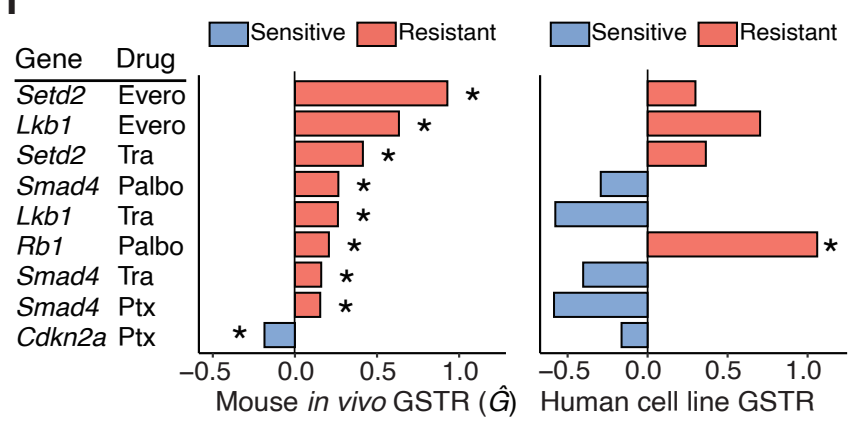

j $\quad$ k

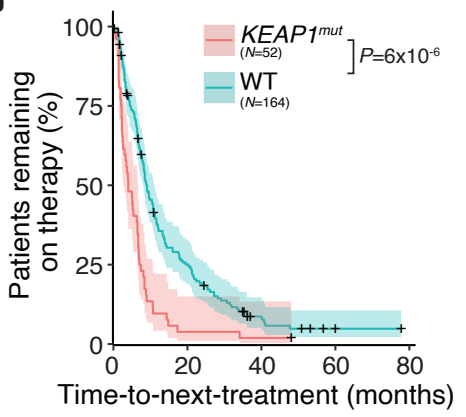

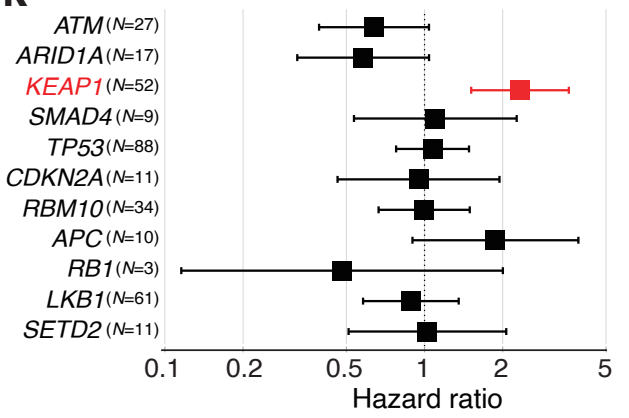

Figure 2. Tuba-seq quantifies genotype-specific therapeutic responses (GSTR) for multiple therapies.

a. Data analysis pipeline to identify GSTR by comparing the relative tumor number (ScoreRTN) and relative geometric mean (ScoreRGM) between tumors containing a tumor suppressor targeting sgRNA and Inert tumors in the untreated and treated mice.

b. The sensitivity and specificity of ScoreRTN estimated from simulations of preassigned drug effect ( $S=0.5$ ) and GSTR (various G). There is no genotype-specific response when $G=0$. $G$ of $-20 \%$ means the tumors with the sgRNA were reduced by an additional $20 \%$ in size.

c. The sensitivity and specificity of ScoreRGM estimated from the same simulation as in b.

d. Likely mechanism of action for monotherapies used for treatments.

e. Timeline of the experiment. Tumors were initiated in $K T ; H 11^{L L L-C a s 9}$ mice with the barcoded Lenti-sgTSPool/Cre. Three weeks of treatment was initiated after 15 weeks of tumor growth.

f-h. G calculated from the inverse variance weighted average of ScoreRTN and ScoreRGM for the pharmacogenomic mapping experiment (f), negative control experiment in $K T$ mice $(\mathbf{g})$ and palbociclib repeat experiment $(\mathbf{h})$. Stars represent significant cases.

i. Comparison of our identified GSTRs with those from the Genomics of Drug Sensitivity in Cancer (GDSC) database. Stars represent significant cases.

j. Kaplan-Meier curve (with 95\% confidence interval in shading) of time-to-next-treatment (months) for patients with or without KEAP1 mutations with metastatic oncogenic KRAS-driven lung adenocarcinoma to platinum-containing chemotherapy. The number of patients in each group is shown. $P$-values were calculated from the Mantel-Haenszel test.

k. Responses of patients with metastatic oncogenic KRAS-driven lung adenocarcinoma to platinum-containing chemotherapy are consistent with KEAP1 inactivation leading to resistance. KEAP1 mutations are significantly correlated with a higher hazard ratio for time-to-next-treatment. 
bioRxiv preprint doi: https://doi.org/10.1101/2020.01.28.923912; this version posted January 29, 2020. The copyright holder for this preprint (which was not certified by peer review) is the author/funder. All rights reserved. No reuse allowed without permission.

Li and Lin et al.

a

Paired-end sequencing of the sgID-BC amplicon using Hi-Seq2500

$\downarrow$

Extract the sgID-BC region

keeping only those reads that match

perfectly in forward and reverse direction

Keep barcodes with 2 or more reads

$\downarrow$

Remove all "tumors" with barcodes that are within 2 nucleotides of any larger

tumor in that mouse with the same sgID

$\downarrow$

Determine the \# of reads with each barcode and calculate the absolute \# of neoplastic cells by comparing to the \# of reads from Spike-in controls

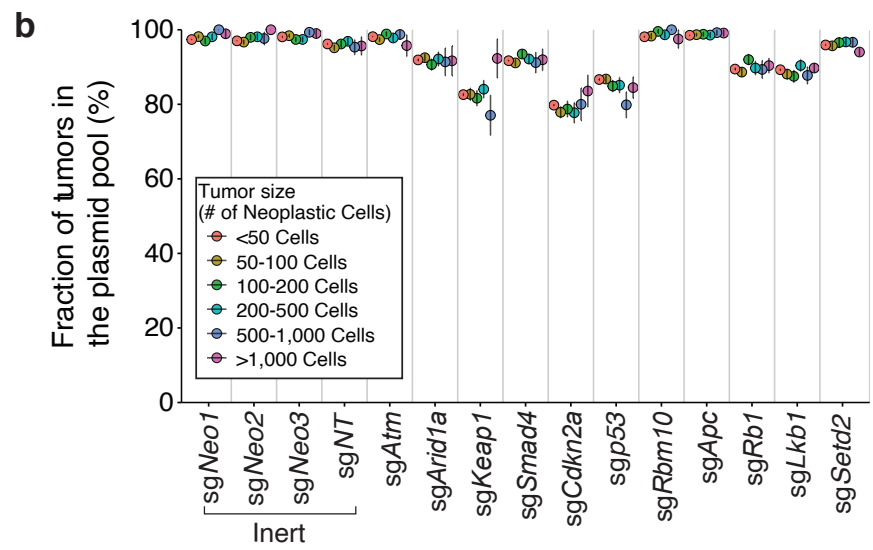

C

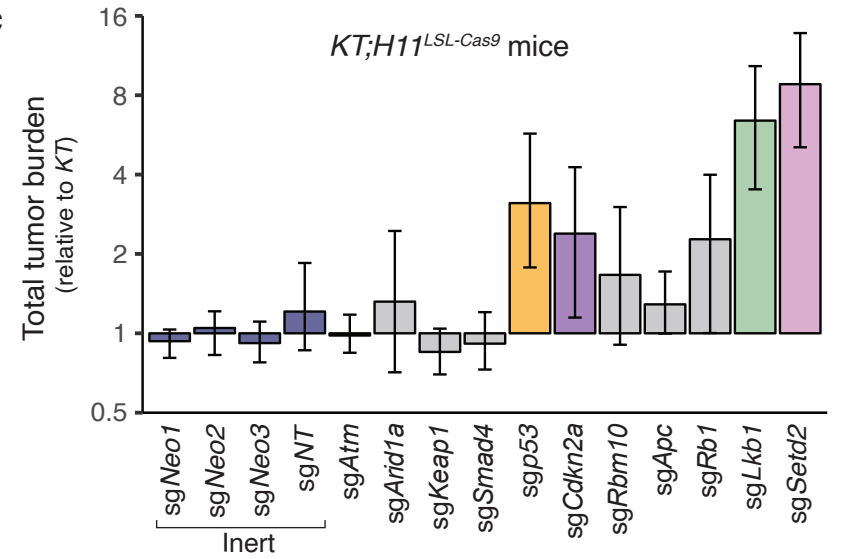

e

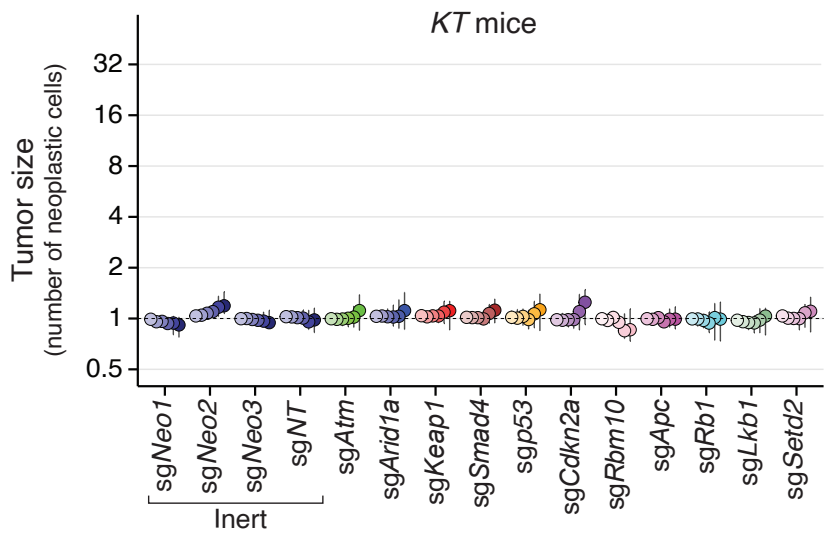

d

$\begin{array}{cc}\text { KT } & \text { KT;H11 } 1_{\text {LSL-Cas9 }}^{\text {LN Mean }} \\ \begin{array}{l}\text { LN Mean } \\ \text { +/- SD p-value } \\ +/ \text { - SD }\end{array} \text {-value }\end{array}$

$\left[\begin{array}{ccccc}\text { sgNeo1 } & 0.95+/-0.05 & 0.07 & 0.98+/-0.05 & 0.70\end{array}\right.$

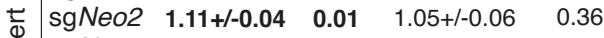

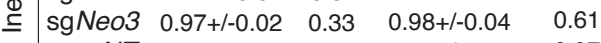

$\begin{array}{lllll}\operatorname{sgNT} & 1.01+/-0.04 & 0.82 & 1.01+/-0.10 & 0.97\end{array}$

$\begin{array}{lllll}\text { sgAtm } & 1.02+/-0.05 & 0.64 & 0.92+/-0.07 & 0.29\end{array}$

$\begin{array}{lllll}\text { sgArid1a } & 1.05+/-0.06 & 0.38 & 1.05+/-0.12 & 0.75\end{array}$

$\begin{array}{lllll}\text { sgKeap1 } & 1.05+/-0.04 & 0.23 & 1.11+/-0.16 & 0.43\end{array}$

$\begin{array}{lllll}\text { sgSmad4 } & 1.04+/-0.05 & 0.41 & 1.09+/-0.09 & 0.26\end{array}$

$\begin{array}{lllll}\text { sgp53 } & 1.02+/-0.06 & 0.71 & 1.16+/-0.11 & 0.10\end{array}$

$\begin{array}{lllll}\text { sgCdkn2a } & 1.07+/-0.06 & 0.25 & \mathbf{1 . 3 4 + / - 0 . 1 7} & \mathbf{0 . 0 0 6}\end{array}$

$\begin{array}{lllll}\mathrm{sgRbm} 10 & 0.96+/-0.05 & 0.49 & \mathbf{1 . 5 3 + / - 0 . 3 8}<0.0001\end{array}$

$\operatorname{sgApc} \quad 1.00+/-0.03 \quad 0.94 \quad \mathbf{1 . 2 7 + / - 0 . 1 0} \quad \mathbf{0 . 0 0 4}$

$\begin{array}{lllll}\mathrm{sgRb} 1 & 0.98+/-0.06 & 0.65 & 1.45+/-0.32 & 0.054\end{array}$

sgLkb1 $0.96+/-0.04 \quad 0.17 \quad 6.78+/-2.87<0.0001$

sgSetd2 $1.03+/-0.06 \quad 0.60 \quad 17.8+/-5.25<0.0001$

\section{Supplementary Figure 1. Optimization of Tuba-seq increases the resolution and precision of tumor analyses}

a. Overview of our new Tuba-seq analysis pipeline for calling sgID-BC from sequencing data and determining the number of neoplastic cells in each tumor (tumor size).

b. Fractions of tumor sgID-BC region recovered in the plasmid pool across multiple size ranges. By sequencing the sgID-BC region in the Lenti-sgRNA/Cre plasmids, we define a high confidence list of barcodes that are present in each sgID-BC region. If spurious tumor remains, the identified smaller tumors in $K T$;Cas 9 mice will less likely be uncovered in the plasmid pool compared with larger tumors.

However, we find that equivalent proportions of tumors at all sizes are present in the plasmids pool. Note that not all barcodes found in tumors are found in the sequenced plasmids pool because the sequencing depth of the plasmid pool was insufficient to uncover all barcodes in the plasmid pools.

c. Total tumor burden in $K T$;Cas9 mice with Lenti-TSPool/Cre-initiated tumors relative to expected tumor burden calculated from $K T$ mice. Error bars show $95 \%$ percent confidence intervals. Note that targeting p53 enables the generation of rare very large tumors, hence the tumor-suppressive effect of p53 is easily identified by this metric (which aggregates all cancer cells), while the $95^{\text {th }}$ percentile of tumor size distribution and LN mean are much less dramatic.

d. The relative $L N$ mean for tumors with each sgRNA in $K T$ and $K T ; H 11^{L L L-C a s 9}$ mice 18 weeks after tumor initiation normalized to that of all Inert tumors. Bootstrapped p-values are shown. $P$-values $<0.05$, and their corresponding means are in bold.

e. The relative size of tumors initiated with each Lenti-sgRNA/Cre vector in $K T$ mice 18 weeks after tumor initiation with Lenti-sgTSPool/Cre. Relative size of tumors at the indicated percentiles within the distribution represent merged data from 4 mice. $95 \%$ confidence intervals are shown. 
Li and Lin et al.

a

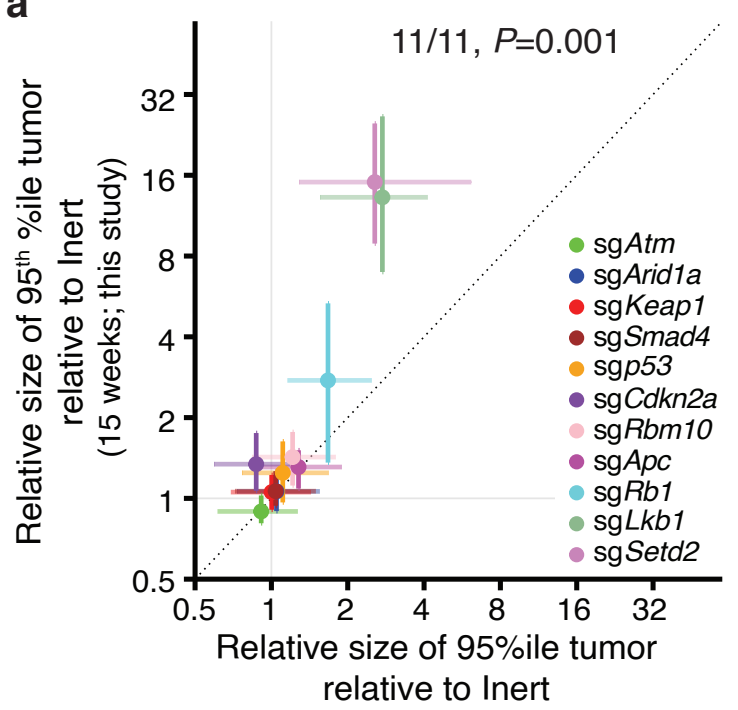

(15 weeks; Rogers et al., 2017)

C

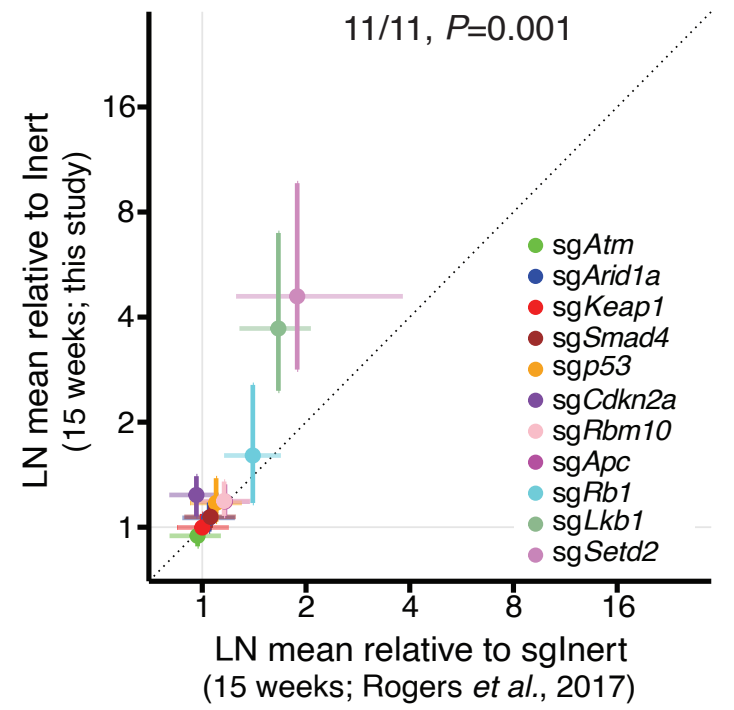

b

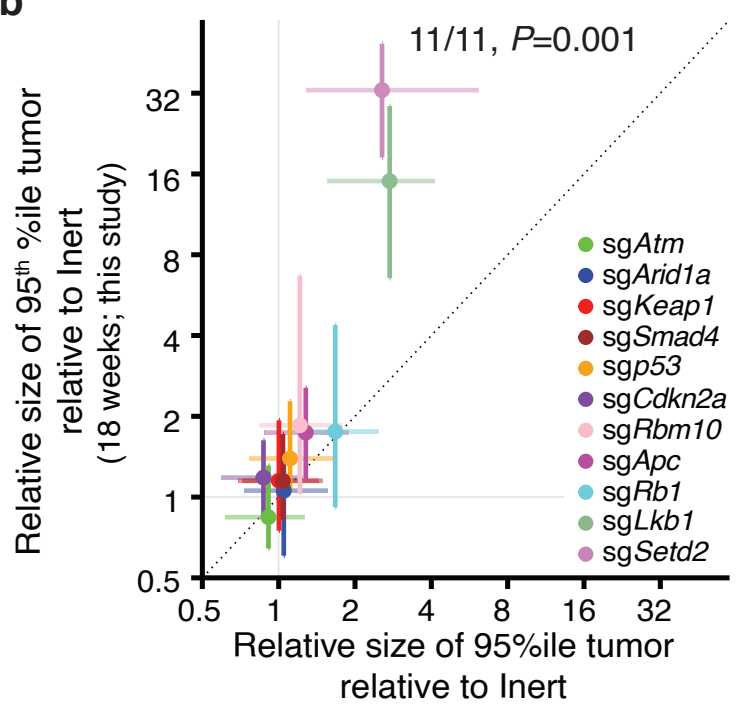

(15 weeks; Rogers et al., 2017)

\section{d}

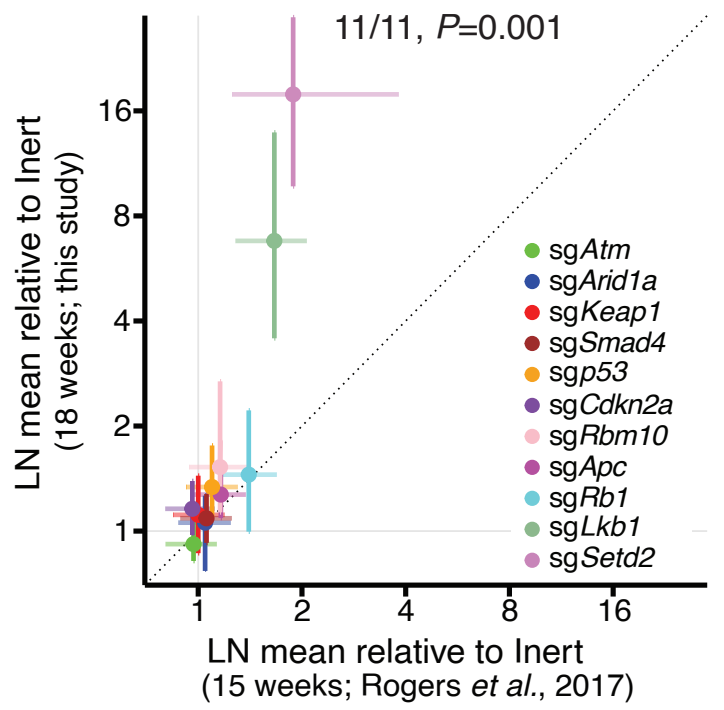

Supplementary Figure 2. Optimization of Tuba-seq increases the resolution and precision of tumor analyses a,b. Comparison of the relative $95^{\text {th }}$ percentile tumor sizes (size of $95^{\text {th }}$ percentile sgTS tumor/size of $95^{\text {th }}$ percentile Inert tumor) of each genotype between the previous data (Rogers et al., 2017) and the current data (this manuscript). Error bars show the $95 \%$ confidence interval. Current data from tumors 15 weeks (a) and 18 weeks (b) after tumor initiation are shown.

c,d. Comparison of the relative LN mean (LN mean of sgTS tumor/LN mean of Inert tumors) of the tumors of each genotype between the previous data (Rogers et al., 2017) and the current data (this manuscript). Error bars show the $95 \%$ confidence interval. Current data from tumors 15 weeks (c) and 18 weeks (d) after tumor initiation is shown. In each panel, Error bars show the $95 \%$ confidence intervals. Dash lines represent equal detected magnitudes of tumor suppression across studies. $11 / 11$ means 11 out of 11 the assayed genotypes showed a higher magnitude in current studies, and $P$-values are calculated from the sign-test of the difference in magnitudes for each metric between this study and the previous study. 
bioRxiv preprint doi: https://doi.org/10.1101/2020.01.28.923912; this version posted January 29, 2020. The copyright holder for this preprint (which was not certified by peer review) is the author/funder. All rights reserved. No reuse allowed without permission.

Li and Lin et al.

a
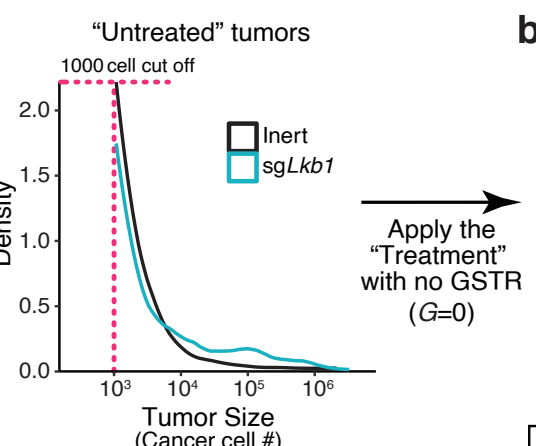

b

"Treated" tumors
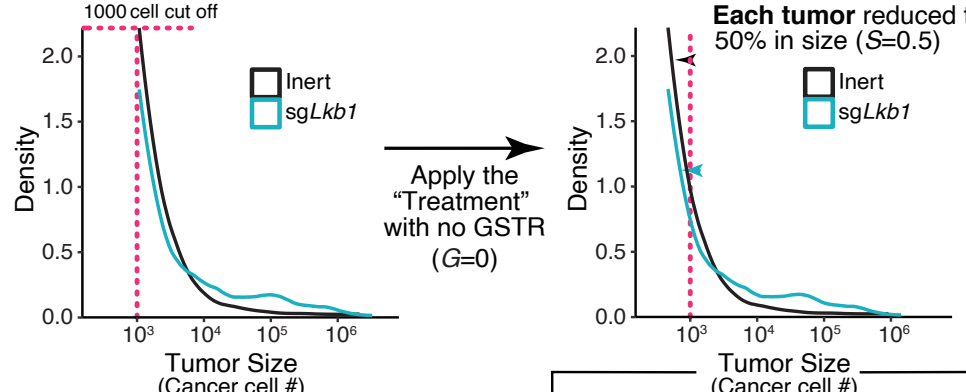

C
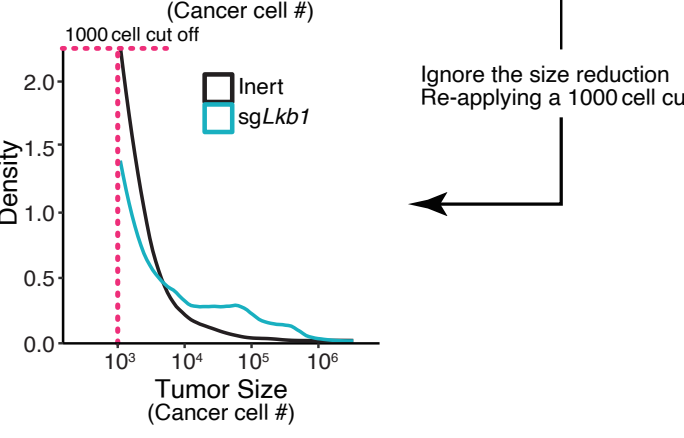

Re-applying a 1000 cell cutoff

$\mathbf{e}$

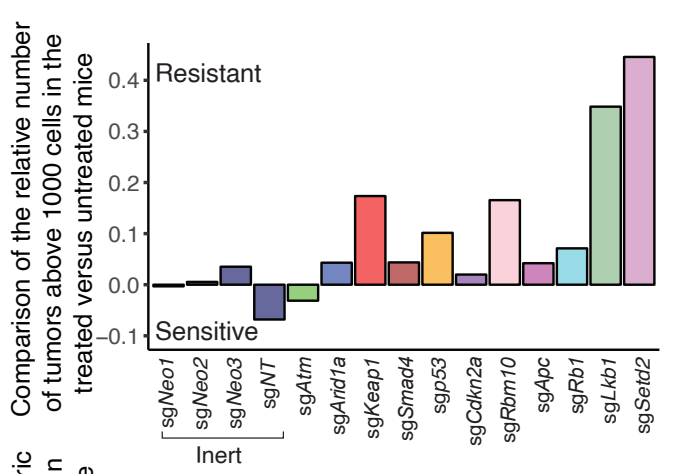

f

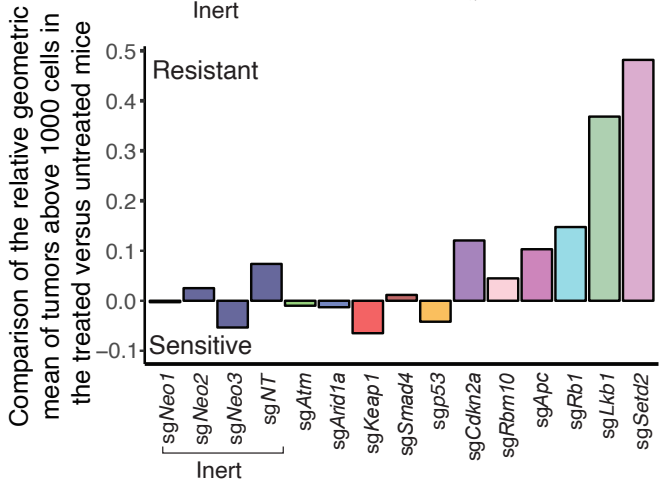

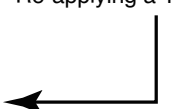

g

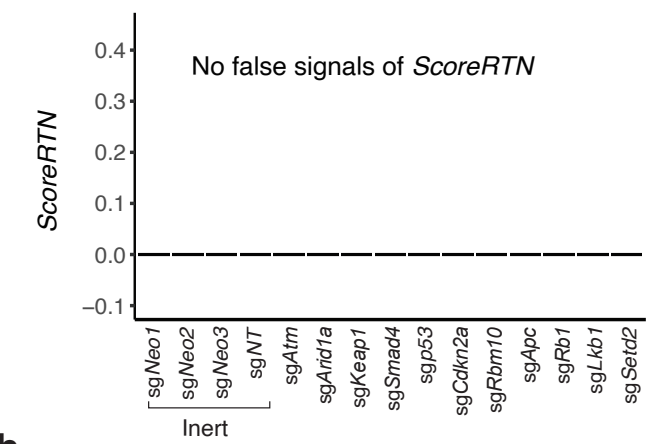

h

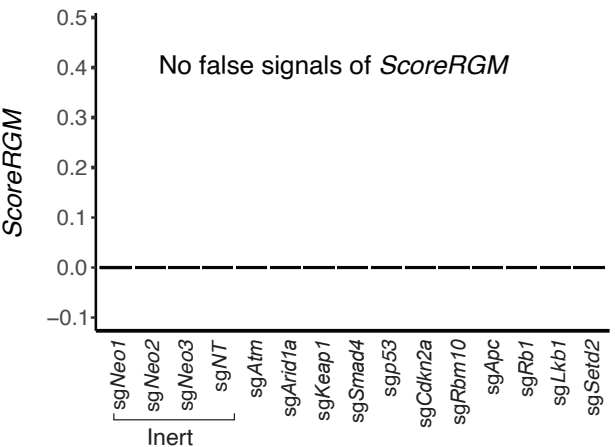

Supplementary Figure 3. The intuitive approach of comparing tumors above a constant cutoff between the untreated and treated mice generates aberrant signals of Genotype-specific treatment responses (GSTRs)

a. Tumor size distributions of "untreated" Inert and sgLkb1 tumors were pooled from 5 KT;Cas9 mice 18 weeks after tumor initiation for all simulations. Tumors with more than 1,000 neoplastic cells are plotted. Inert tumors are tumors containing sgNeo1, $\mathrm{sgNe02}$, sgNeo3, or sgNT. Targeting $L k b 1$ changes the overall shape of the tumor size distribution.

b. Tumor size distributions of "treated" Inert and sg $L k b 1$ tumors were generated by reducing the size of every tumor to $50 \%$ $(S=0.5)$, assuming each cancer cell was killed by the drug with a $50 \%$ probability $(G=0)$.

c. After re-applying a 1000 cell cut off, the new tumor size distributions of Inert and sgLkb1 tumors are noticeably different from those before the size reduction in panel a.

d. To account for tumor size reduction, we need to adjust the cutoff in the treated group by the estimated size reduction due to treatment.

e,f. Comparing the relative tumor number (e) and relative geometric mean (f) (normalized to the corresponding Inert tumors) between the "untreated" and "treated" tumors with each sgID by taking the $\log _{2}$ ratio of the metric in the treated mice over that of the untreated mice for tumors with each sgID. The values are non-zero. Therefore, this intuitive approach is incorrect.

$\mathbf{g , h}$. After correcting for the overall tumor size reduction by the treatment, no false signals of ScoreRTN (g) and ScoreRGM (h) were generated when comparing the relative tumor number and relative geometric mean between the "untreated" and "treated" mice. 
bioRxiv preprint doi: https://doi.org/10.1101/2020.01.28.923912; this version posted January 29, 2020. The copyright holder for this preprint (which was not certified by peer review) is the author/funder. All rights reserved. No reuse allowed without permission.

Li, Lin et al.

a

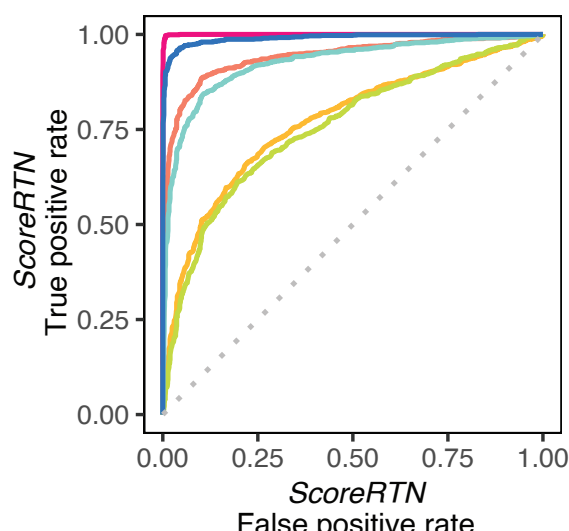

False positive rate

b

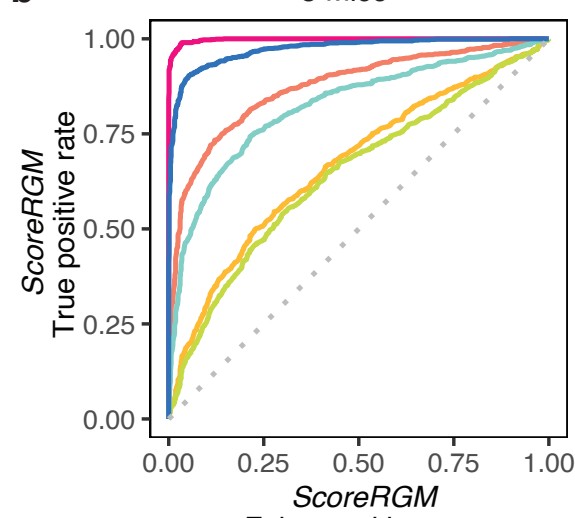

False positive rate

C

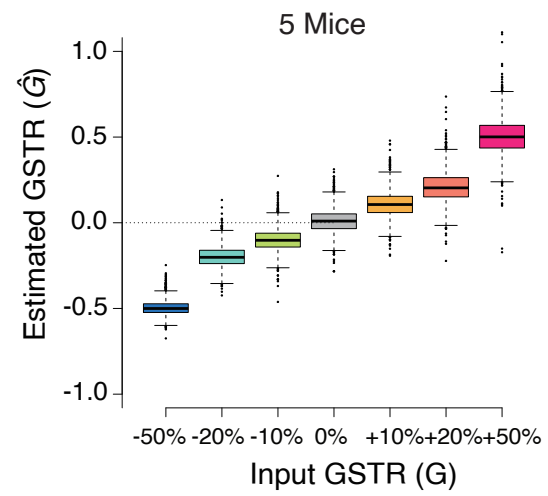

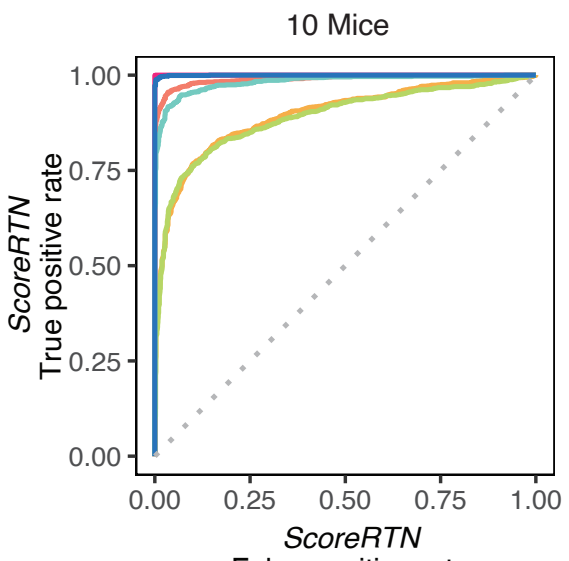

False positive rate

10 Mice

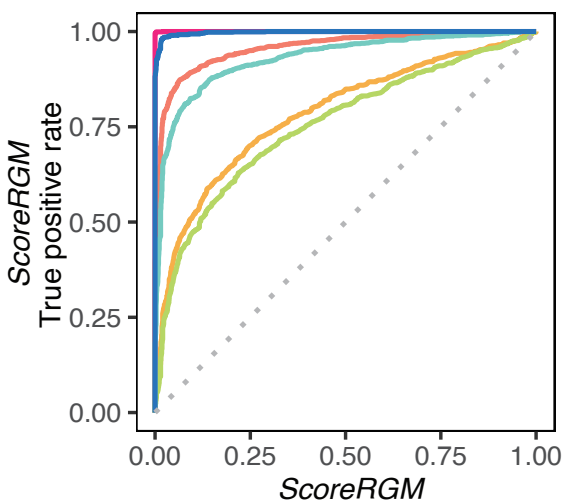

False positive rate

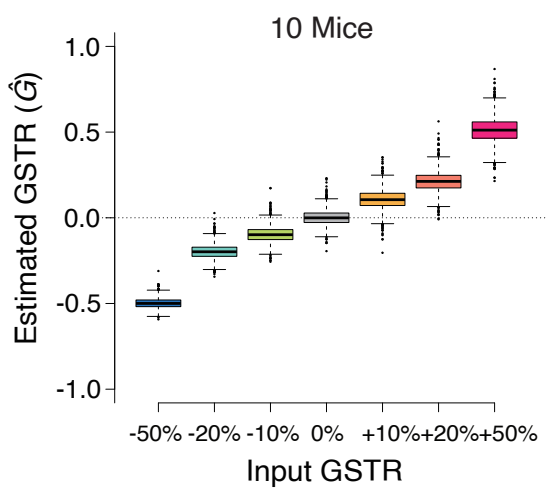

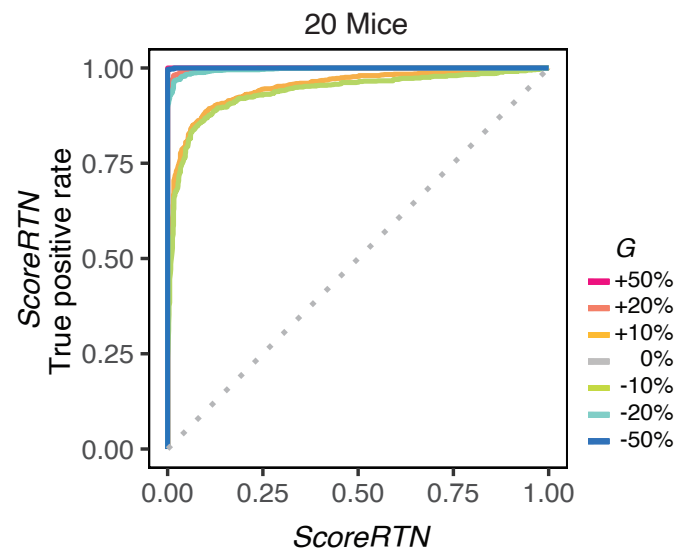

False positive rate

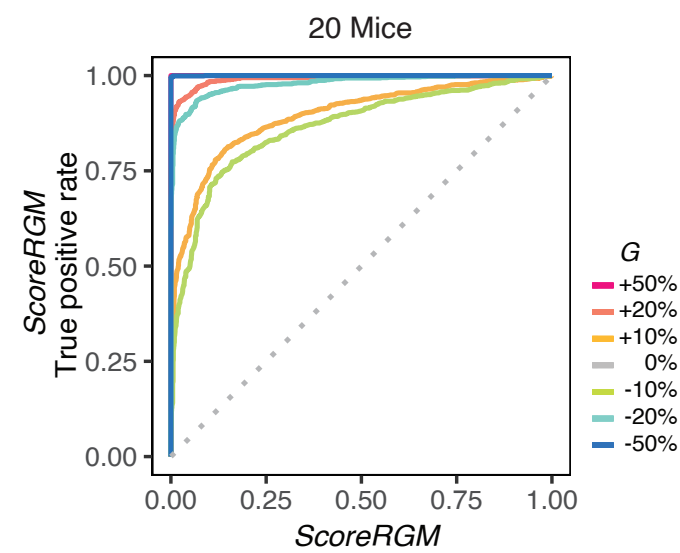

False positive rate

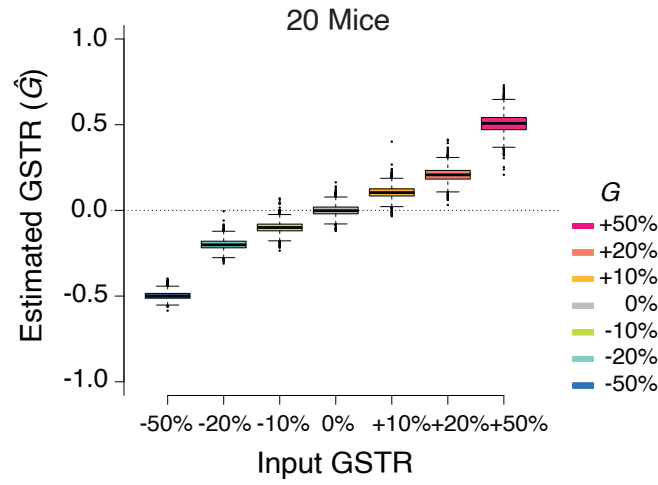

\section{Supplemental Figure 4. Power analysis for various sample sizes.}

a. The sensitivity and specificity of ScoreRTN estimated from simulation of preassigned drug effect ( $S=0.5)$ and various input GSTR (G) when various numbers of mice were used in the treated and untreated group (for example, "10 mice" means that 10 untreated and 10 treated mice were sampled from 8 untreated mice from the pharmacogenomic mapping experiment with replacement for simulation, respectively). The increase in sample size or input $\operatorname{GSTR}(G)$ leads to a higher power.

b. The sensitivity and specificity of ScoreRGM were estimated with the same parameter setting as in a.

c. Estimated GSTR $(\hat{G})$ by combining ScoreRTN and ScoreRGM from the above simulations with various input GSTR (G). The estimated GSTRs are unbiased and were more accurate with larger sample size. 
bioRxiv preprint doi: https://doi.org/10.1101/2020.01.28.923912; this version posted January 29, 2020. The copyright holder for this preprint (which was not certified by peer review) is the author/funder. All rights reserved. No reuse allowed without permission.

Li, Lin et al.

a
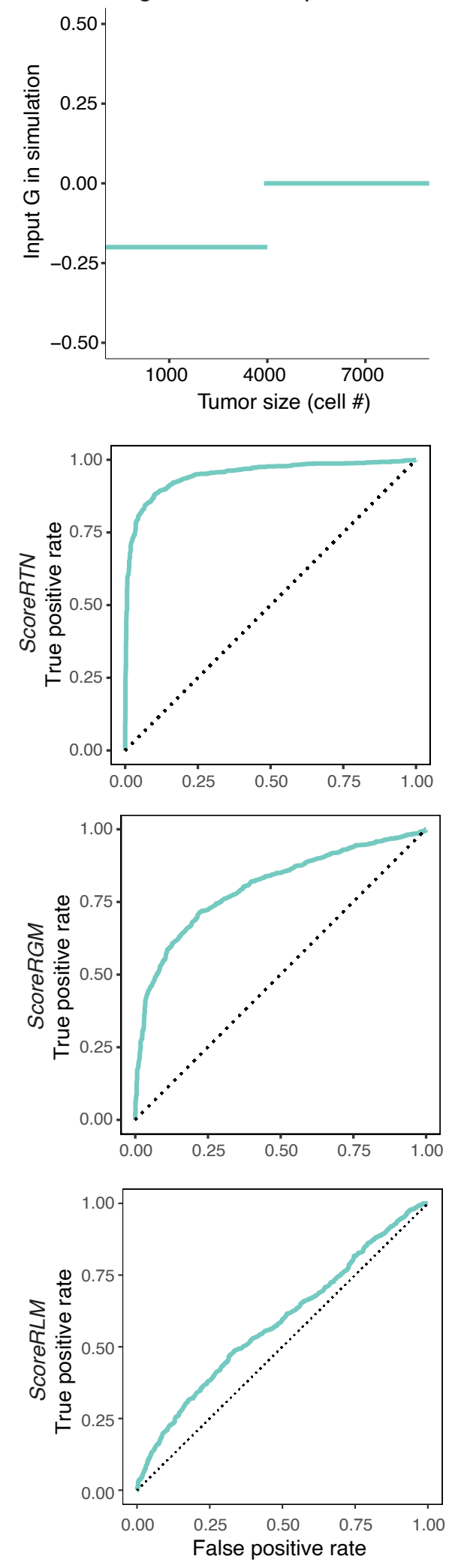

b
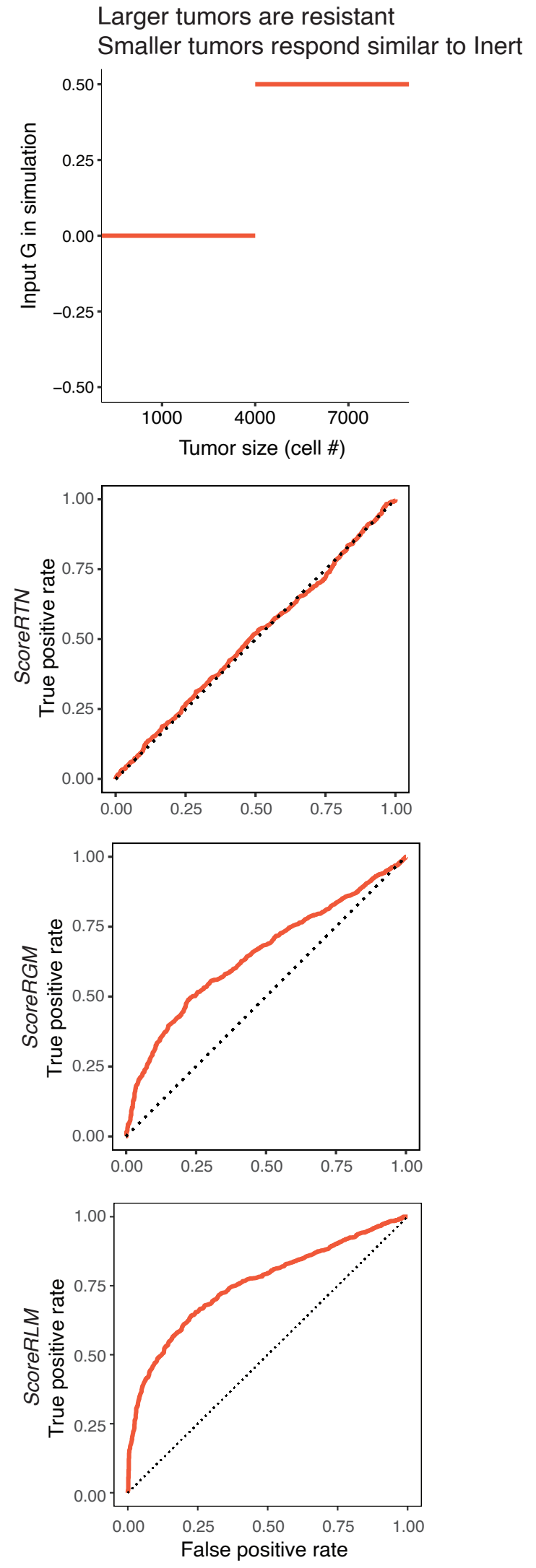

Supplemental Figure 5. Examples of scenarios where one score outperforms the other

a. Five untreated and treated mice were simulated as in Supplementary Fig 4 with $S=0.5$. The top panel shows the preassigned input GSTR $(G)$ on non-Inert tumors, where tumors smaller than 4000 cells showed an additional $20 \%$ reduction in size. When small tumors respond more, ScoreRTN outperforms ScoreRGM in detecting genotype-specific responses.

b. The simulation was the same as panel a except for the preassigned input GSTR. The top panel shows the input GSTR (G), where tumors larger than 4000 cells respond less well to the drug compared with Inert tumors, resulting in tumors being $50 \%$ larger than expected when $G=0$. When large tumors respond less, ScoreRTN is not a sensitive metric to detect genotype-specific responses; however, ScoreRGM retains some power to detect genotype-specific responses. Other summary statistics, such as ScoreRLM that compares the relative LN mean for tumors between the treated and untreated mice, can also be used to identify GSTR. Compared with ScoreRTN and ScoreRGM, ScoreRLM has the lowest power when larger tumors respond similar to Inert tumors, and has the highest power when only larger tumors show GSTR. 
Li, Lin et al.

a

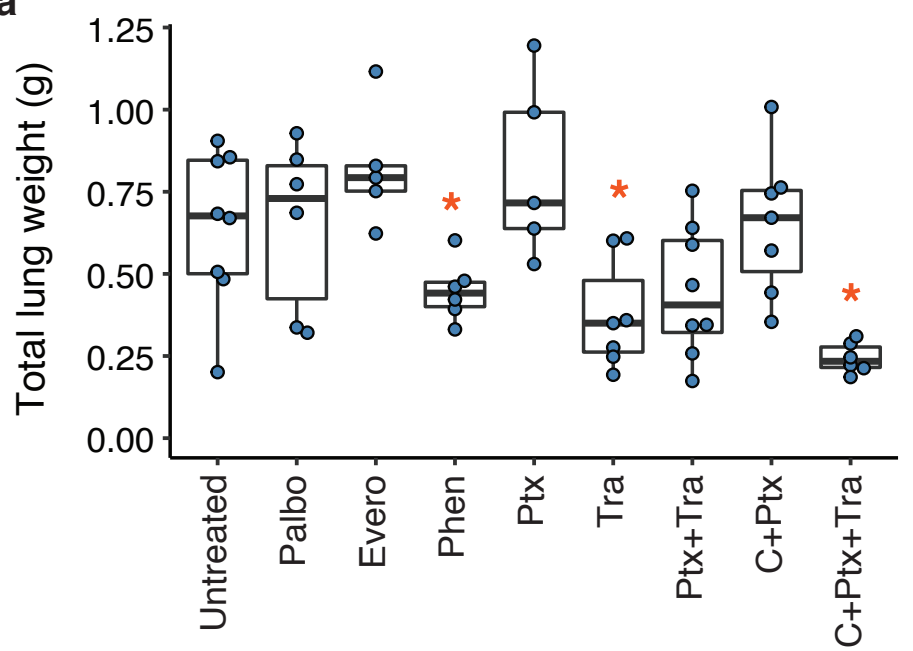

b

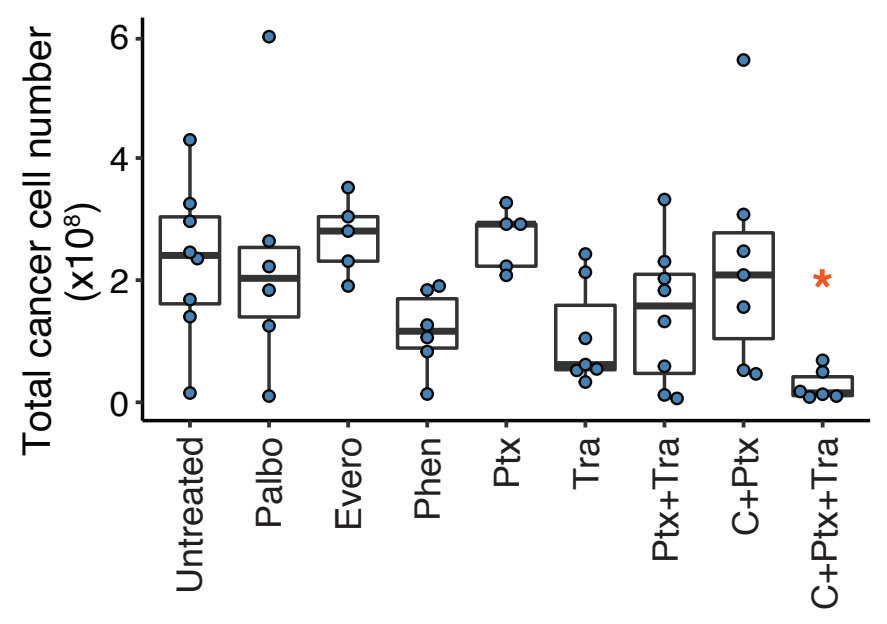

C

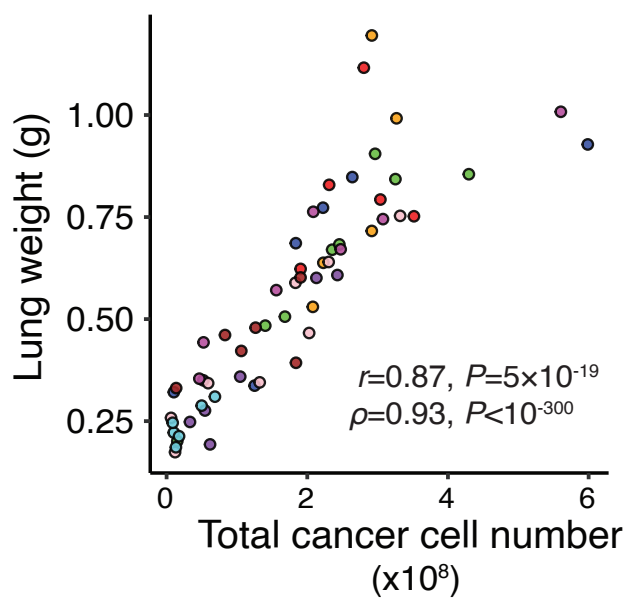

d

Treatment

- Untreated

- Palbo

- Evero

- Phen

- Ptx

- Tra

- Ptx+Tra

- $\mathrm{C}+\mathrm{Ptx}$

- C+Ptx+Tra

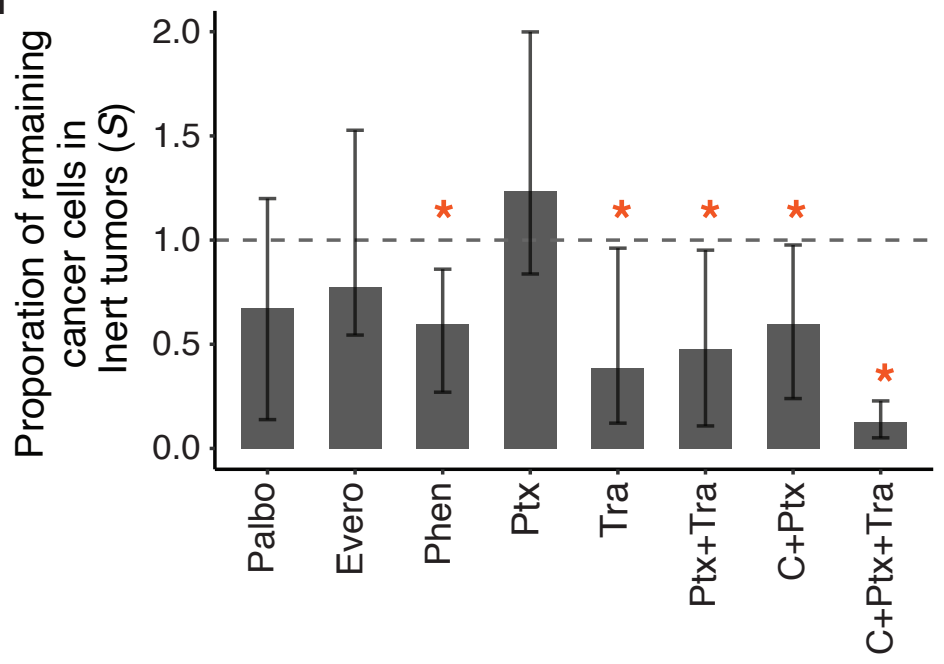

\section{Supplemental Figure 6. Overall treatment responses of mice to therapies}

a. Boxplot showing the effects of treatment on mouse lung weight. Each blue dot is the lung weight of a mouse. Several treatments lead to reduced tumor burden sufficient to dramatically reduce total lung weight. Stars below the name of the treatment indicate significant reductions in lung weight by the treatment $(P<0.05$, Mann-Whitney $U$ test $)$.

b. Boxplot showing the effect of treatment on the total cancer cell number. Each blue dot is the total cancer cell number of a mouse. The total cancer cell number in each lung was determined by converting the number of reads containing a sgID to cell number based on the read number of the three spike-ins with known cell number counts. Stars indicate significant reductions in total cancer cell number by the treatment $(P<0.05$, Mann-Whitney $U$ test).

c. Total lung weight is highly correlated with the total cancer cell number.

d. The proportion of remaining cancer cells for Inert tumors $S$ for each treatment is estimated by matching the distribution of Inert tumors in the treated and untreated mice. Stars indicate significant reductions in the remaining tumor cells ( $P<0.05$ by bootstrap). 
Li, Lin et al.

a

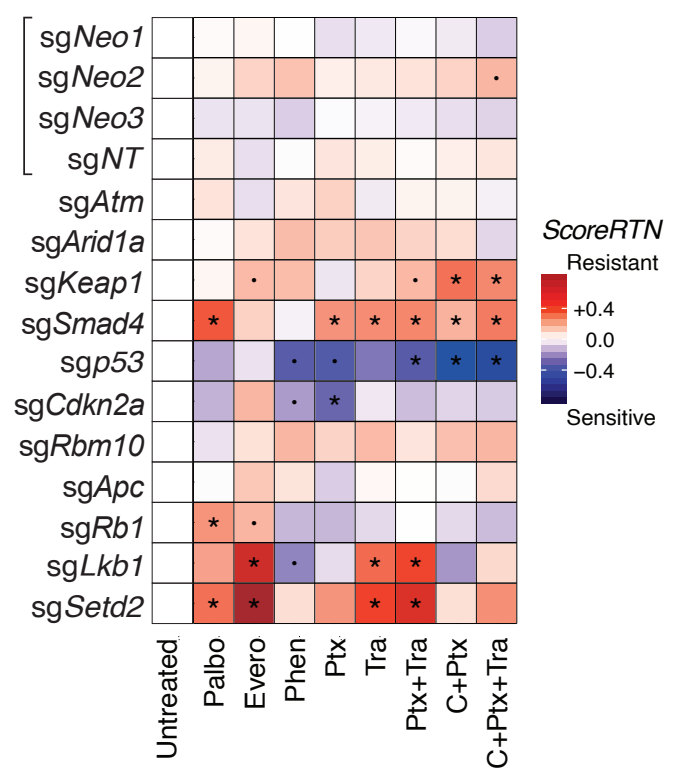

C

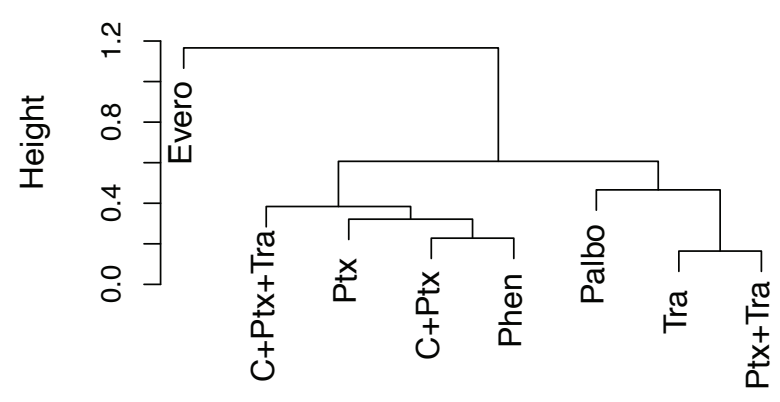

b

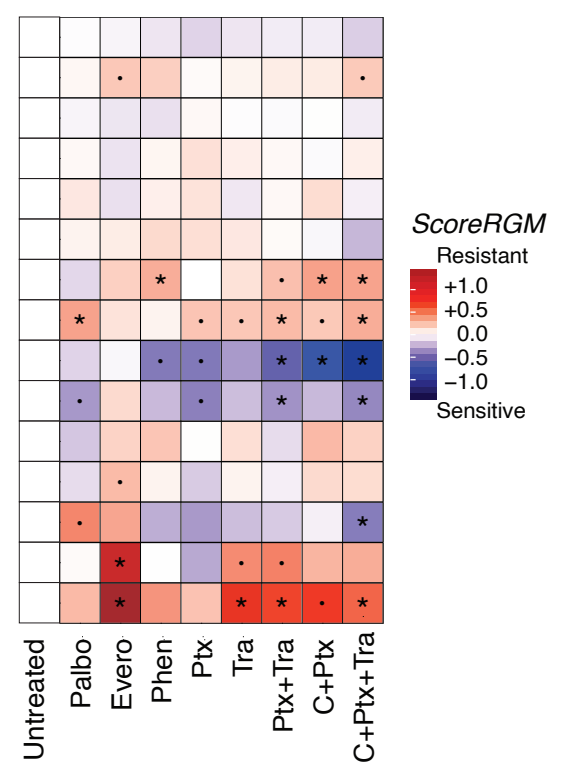

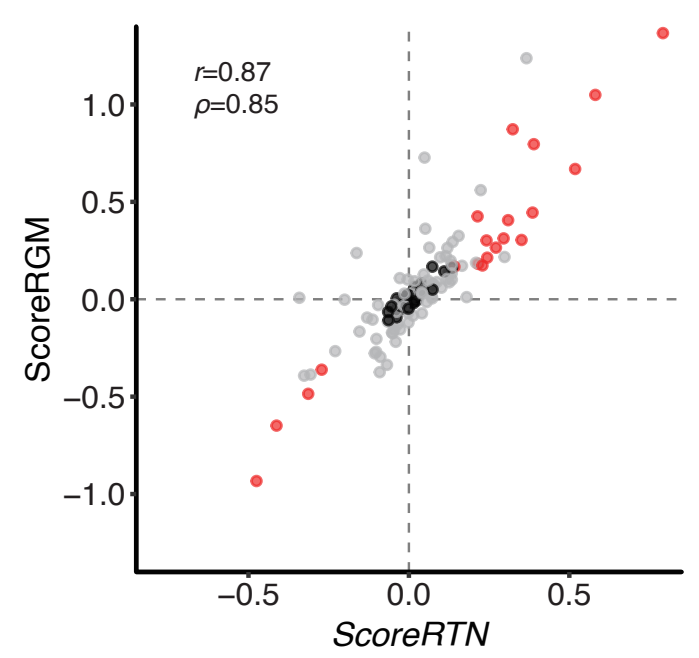

d

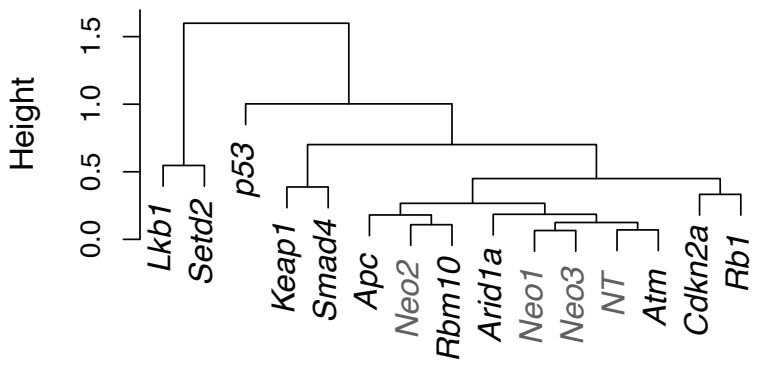

Supplemental Figure 7. ScoreRTN and ScoreRGM for all genotypes across all treatments and experiments

a. Heatmap for ScoreRTN and ScoreRGM for the pharmacogenomic mapping experiment outlined in Figure 2e. "." indicates a marginally significant GSTR $(P<0.1)$, and "*” indicates a significant GSTR $(P<0.05)$. ScoreRTN and ScoreRGM are integrated to generate $\hat{G}$ shown in Figure 2f.

b. ScoreRTN and ScoreRGM are highly correlated. Red dots show interactions that are significant by one score and at least marginally significant by the other score. Gray dots are the rest of GSTR that doesn't fit the above criteria. Black dots show all GSTR for Inerts, all with small magnitudes and non-significant $p$-values.

c. Hierarchical clustering of the treatments in the pharmacogenomic mapping experiment based on $\hat{G}$ with complete linkage. Combo treatments clustered close to their corresponding monotherapies.

d. Hierarchical clustering of the genes in the pharmacogenomic mapping experiment based on $\hat{G}$ with complete linkage. Rb1 and $C d k n 2 a$ are in the same biological pathway and were clustered closely. $L k b 1$ and Setd2 were previously shown to be functionally redundant (Rogers et al. 2018) and were clustered closely. 
bioRxiv preprint doi: https://doi.org/10.1101/2020.01.28.923912; this version posted January 29, 2020. The copyright holder for this preprint (which was not certified by peer review) is the author/funder. All rights reserved. No reuse allowed without permission.

Li, Lin et al.

a

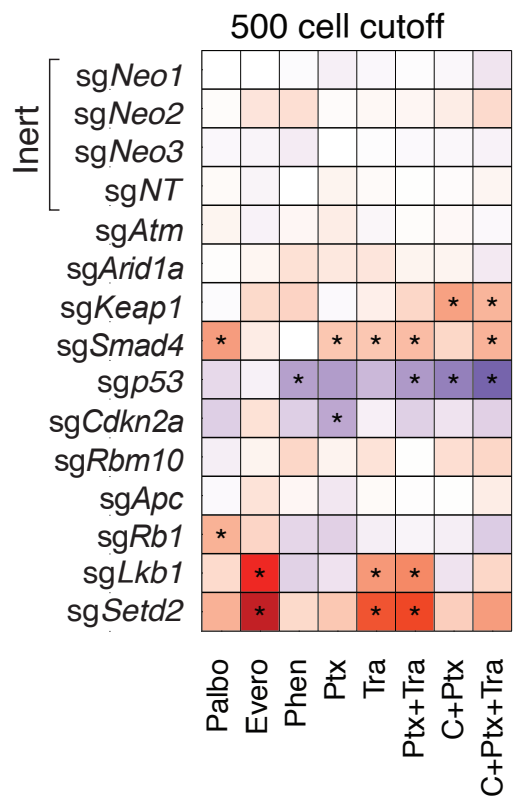

b
1500 cell cutoff

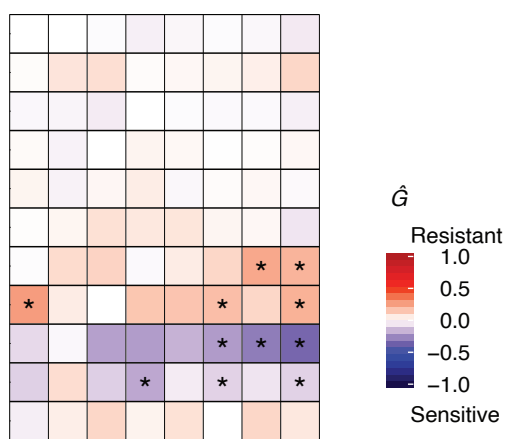

Exclude sgNeo2 from Inert baseline

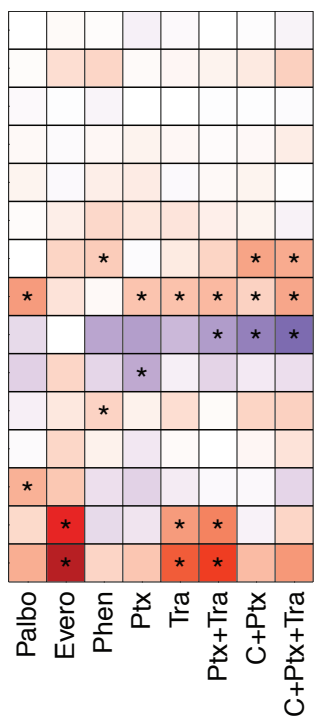

Exclude sgNeo3 from Inert baseline

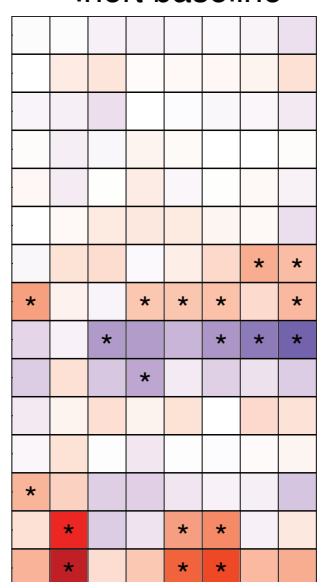

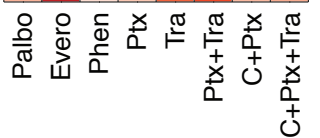

Exclude sgNT from Inert baseline

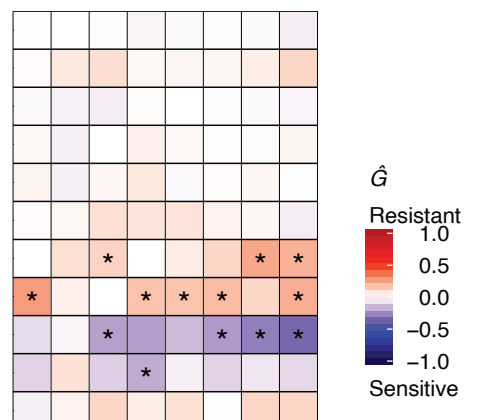

\section{Supplemental Figure 8. Estimates of GSTRs are robust to changes in minimum tumor size cutoff and the composition of the} Inert tumors

a. Throughout most analyses in this manuscript, we include tumors in untreated mice that are calculated to have over 1000 cells (a cutoff of 1000 cells). However, using 500 cells or 1500 cells as the tumor size cutoffs generated very similar results. The correlation between $\hat{G}$ using 500 or 1500 cells as cutoff versus using 1000 cell cutoff was 0.998 and 0.997 for linear correlation, and 0.997 and 0.997 for rank c orrelation, respectively. Compared to using the original 1000 cell cutoff, $100 \%$ and $97.5 \%$ of the GSTR identified by G were in the same di rection, respectively. Among 19 significant GSTRs, 18 and 13 significant GSTRs were reidentified using the 500 and 1500 cell cutoff, re spectively. Thus, the identified genotype-specific therapeutic responses are almost unaltered by using different cell number cutoffs. $\square$ b. Throughout most analyses in this manuscript, we aggregate tumors with sgNeo1, sgNeo2, sgNeo3 and sgNT as the Inert tumors. Excluding any of the Inerts yields similar results. The correlation between $\hat{G}$ leaving out one of the Inerts versus using all four Inerts was over 0.998 for linear correlations and over 0.994 for rank correlations. Over $95.8 \%$ of the Significant $\hat{G}$ results were in the same direction. At least 17 of the 19 significant GSTRs were reidentified in each setting. 
Li, Lin et al.

a

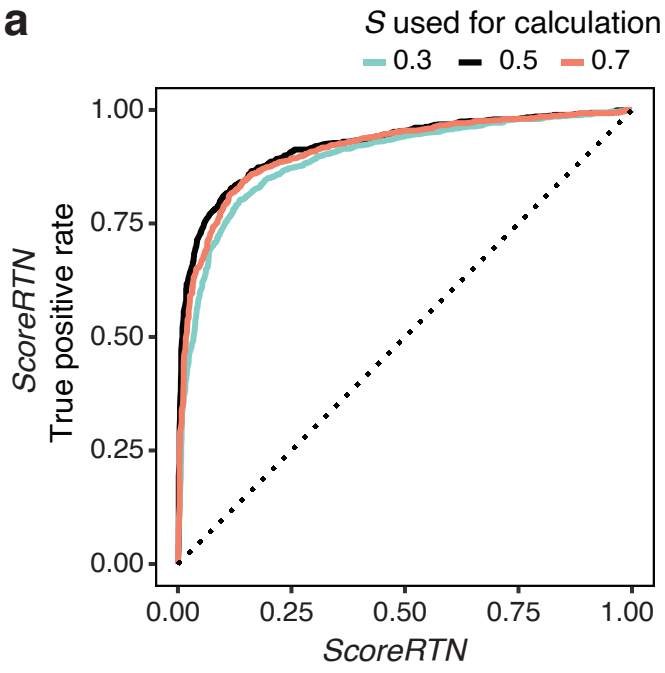

False positive rate

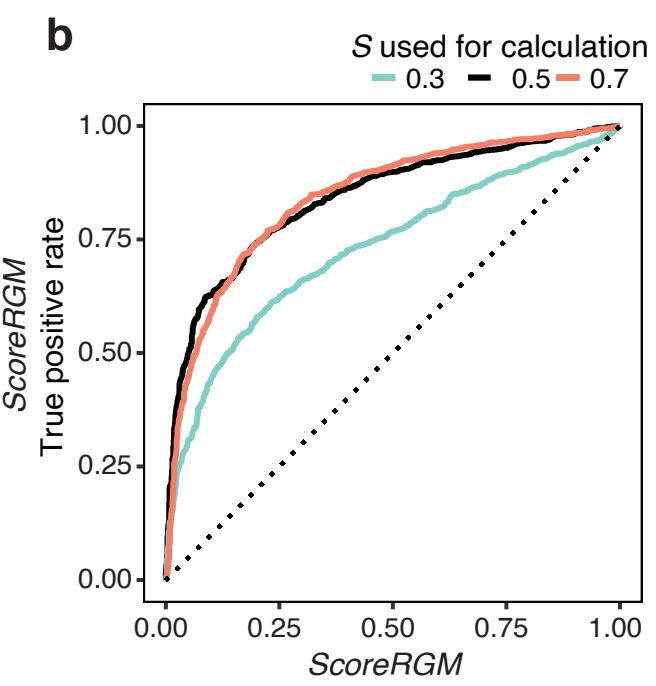

False positive rate

\section{Supplemental Figure 9. Estimates of GSTR by ScoreRTN and ScoreRGM are robust to inaccurate estimation of $S$}

a. The calculation of ScoreRTN and ScoreRGM both depend on our estimate of the overall drug responses (represented as $S$ - the remaining fraction of cancer cells for Inert tumors). In the simulation, we tested the impact of our estimates of $S$ being inaccurate. Instead of estimating the effect size based on observed Inert tumors, we assign $S$ to be a constant value, taking three discrete values $0.3,0.5$, and 0.7 when we know the preassigned drug effect is 0.5 . ROC curves were plotted for detecting GSTRs with Input GSTR $(G)=0.2$. Incorrect estimation of $S$ only slightly impacts the power of ScoreRTN.

b. Similar simulation is performed for ScoreRGM and Incorrect estimation of S only slightly impacts the power of ScoreRGM. 
$\mathrm{Li}$, Lin et al.

a

$\begin{array}{ccc}\begin{array}{c}\text { Tumor } \\ \text { initiation } \\ \text { in } K T \\ \text { mice }\end{array} & \begin{array}{c}\text { Drug } \\ \text { treatment }\end{array} & \text { Analysis } \\ 0 & \text { Time (weeks) } & 21 \\ & \text { Untreated }(n=4) \\ \text { C+Ptx+Tra }(n=5)\end{array}$

b

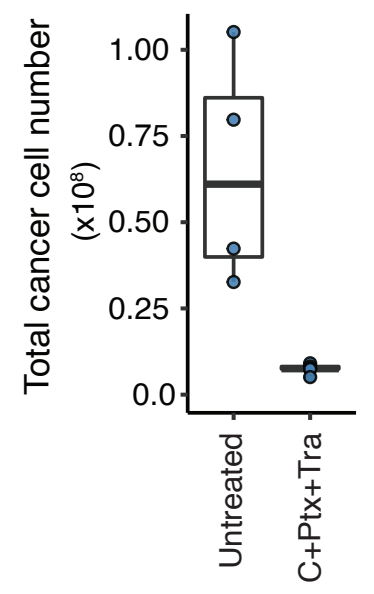

C

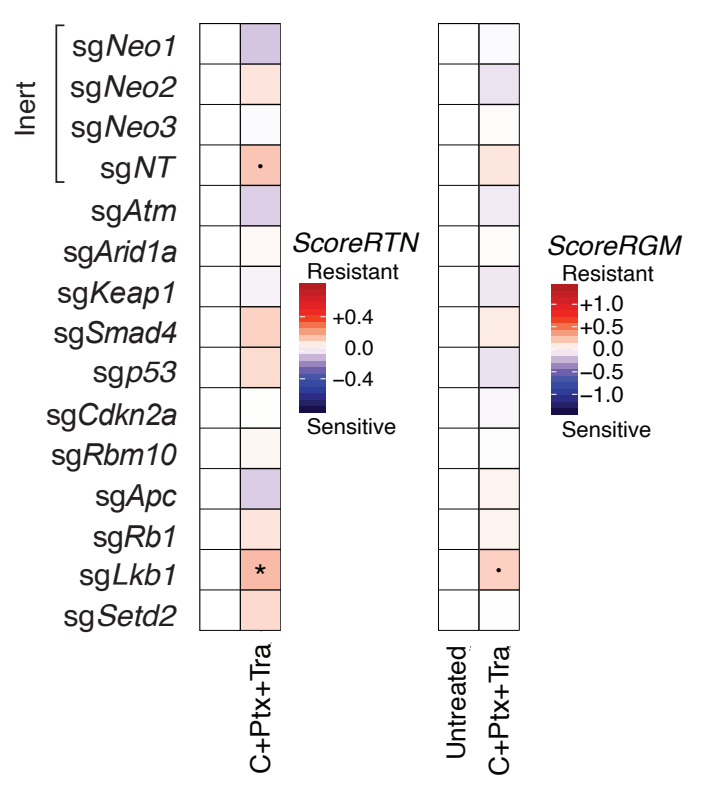

Supplemental Figure 10. Only 1 weak GSTRs were identified in the negative control experiment in $K T$ mice without Cas9. a. Outline of a negative control experiment. In this negative control experiment, we initiated tumors in KT mice (which lack Cas9) with Lenti-TS ${ }^{\mathrm{Poo} /} / \mathrm{Cre}$ followed by a 6-week treatment with Carboplatin+Paclitaxel+Trametinib (C+Ptx+Tra). We chose this treatment, and prolonged treatment time, to generate maximal tumor responses which would most stringently test our analytical models. Given that the mice lack Cas9, all sgRNA are functional Inert, and we expected to find no differences in responses of tumors with different sgRNAs. The number of mice in each group is indicated.

b. Boxplot showing the effect of treatment on the total cancer cell number. Each blue dot is the total cancer cell number of a mouse. The total cancer cell number in each lung was determined by converting the number of reads containing a sglD to cell number based on the read number of the three spike-ins with known cell number counts. The total cancer cell numbers were significantly different across the two groups.

c. Heatmap for ScoreRTN and ScoreRGM for the negative control experiment. "." indicates a marginally significant GSTR $(P<0.1)$, and "*" indicates a significant GSTR $(P<0.05)$. Note the relatively low magnitudes of estimated effect relative to those shown in Supplementary Figure 7a. ScoreRTN and ScoreRGM are integrated to generate $\hat{G}$ shown in Figure $2 \mathrm{~g}$. Apart from tumors with sgLkb1 showing significant and marginally significant GSTRs with small magnitudes by ScoreRTN and ScoreRGM, respectively, other genes were non-significant when comparing the $\mathrm{C}+\mathrm{Ptx}+$ Tra treated mice to untreated $K T$ mice. 
bioRxiv preprint doi: https://doi.org/10.1101/2020.01.28.923912; this version posted January 29, 2020. The copyright holder for this preprint (which was not certified by peer review) is the author/funder. All rights reserved. No reuse allowed without permission.

Li, Lin et al.

a
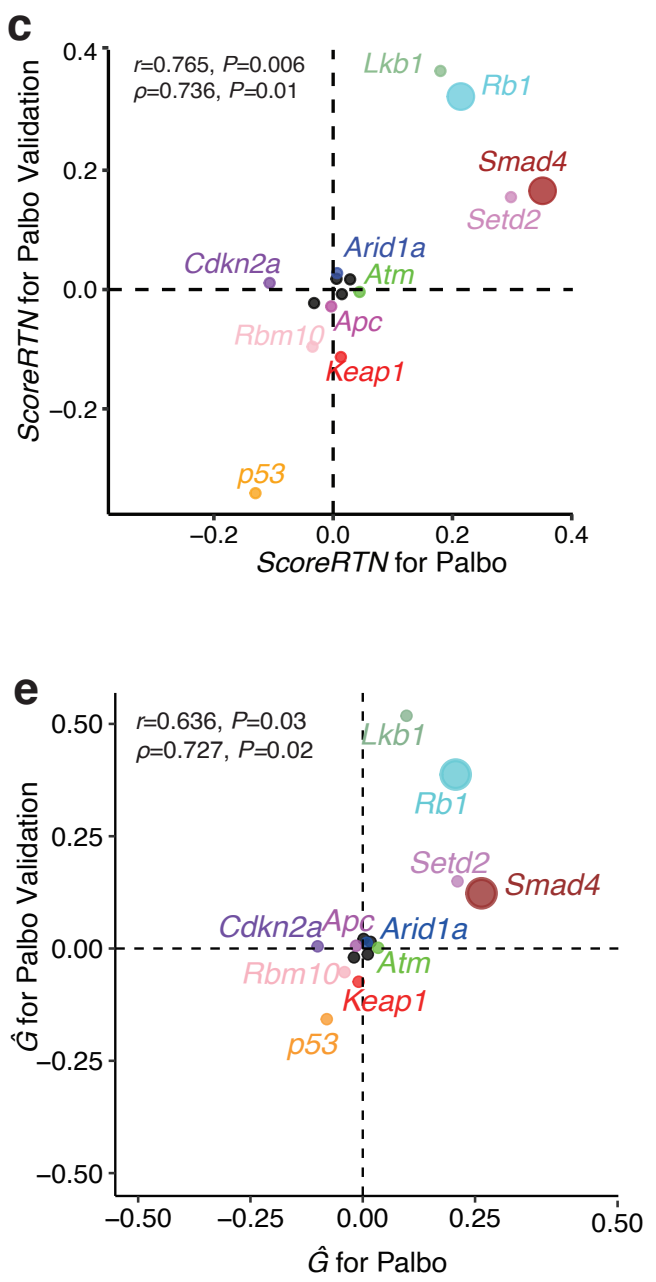

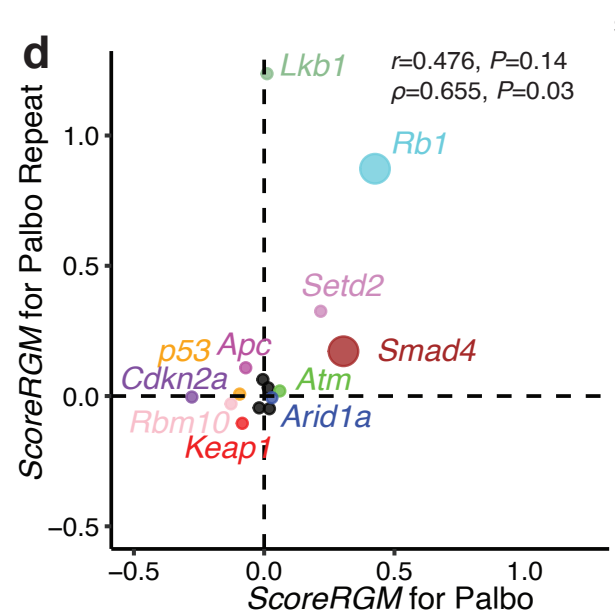

b

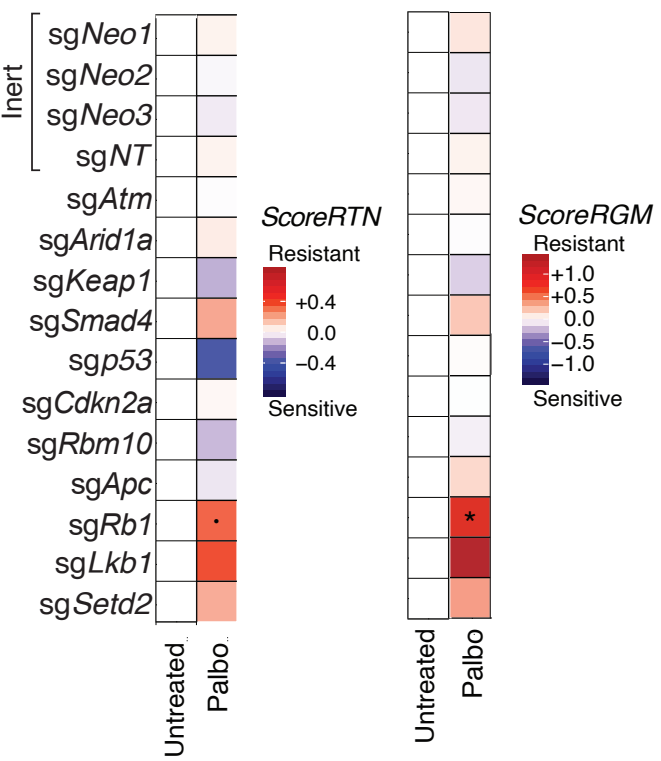

$R b 1 \%$

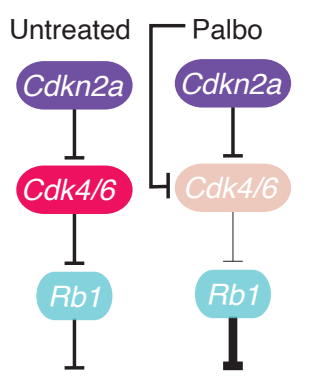

cell cycle

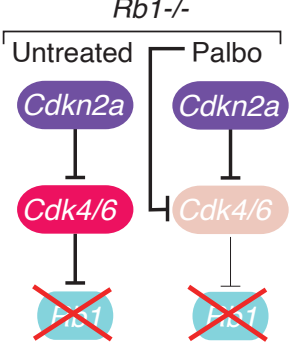

cell cycle cell cycle cell cycle
Cdkn2a-/-

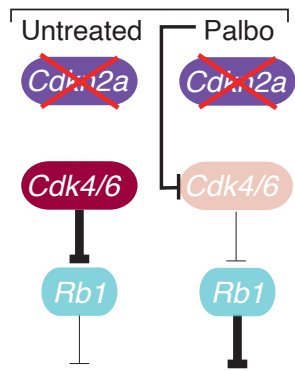

cell cycle

\section{Supplemental Figure 11. GSTR to Palbociclib identified in a repeat experiment.}

a. Outline of the replicate experiment to identify GSTR to Palbociclib (Palbociclib repeat experiment).

b. Heatmap for ScoreRTN and ScoreRGM for the Palbociclib repeat experiment. "." indicates a marginally significant GSTR $(P<0.1)$, and

“*” indicates a significant GSTR $(P<0.05)$. ScoreRTN and ScoreRGM are integrated to generate $\hat{G}$ shown in Figure $2 \mathrm{~h}$.

c,d,e. Across two independent experiments of palbociclib, the identified genotype-specific therapy responses (GSTRs) were recaptured for both ScoreRTN (c), ScoreRGM (d), and $\hat{G}(\mathbf{e})$. Large dots indicate significant GSTRs in at least one experiment, and small dots indicate that GSTR was significant in neither experiment. Pearson's $(r)$ and Spearman's correlations $(\rho)$ were calculated.

f. Palbociclib works as a Cdk4/6 inhibitor. Rb1 as the downstream effector is expected to be resistant to Palbociclib. Cdkn2a functions as a tumor suppressor upstream in the same pathway; therefore, the growth advantage conferred by the inactivation of $C d k n 2 a$ will be removed by the Palbociclib treatment, showing drug sensitivity. While $R b 1$-inactivation lead to significant resistance in both experiments, Cdkn2a-inactivation lead to marginally significant sensitivity for both ScoreRTN and ScoreRGM in the pharmacogenomic mapping experiment. Darker color intensities of Cdk4/6 represents higher activity. 
bioRxiv preprint doi: https://doi.org/10.1101/2020.01.28.923912; this version posted January 29, 2020. The copyright holder for this preprint (which was not certified by peer review) is the author/funder. All rights reserved. No reuse allowed without permission.

$\mathrm{Li}$, Lin et al.

a

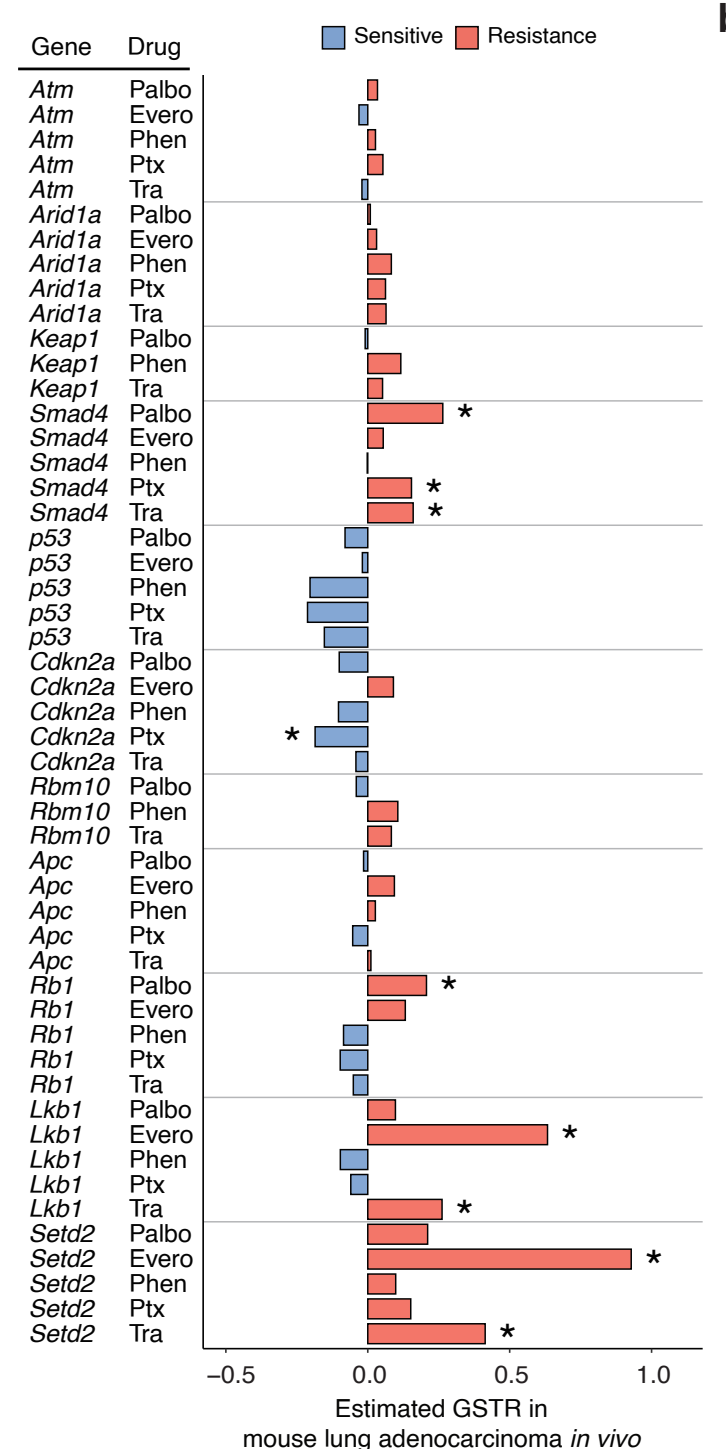

mouse lung adenocarcinoma in vivo b

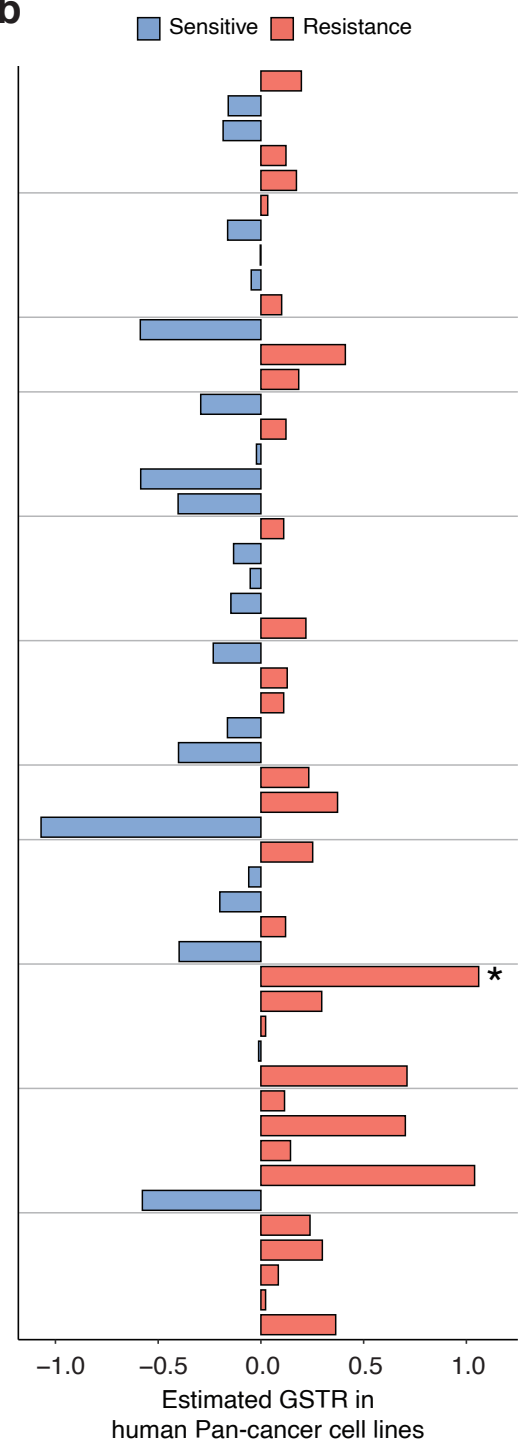

C

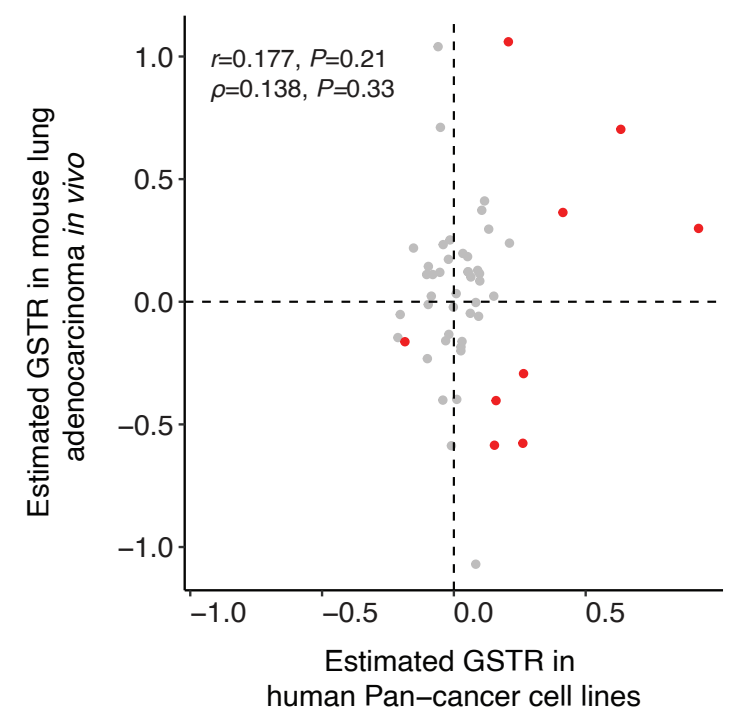

Supplemental Figure 12. Comparison with the GDSC human cell line database

a. GSTR estimated from our study from mouse lung tumors in vivo.

b. GSTR reported by GDSC for human PAN cancer cell lines. Genes are in the same order as a.

c. Correlation between GSTR estimated in our study and the cancer cell line study. The significant GSTRs in our study are highlighted in red. 
$\mathrm{Li}$, Lin et al.

a Everolimus (mTOR inhibitor)

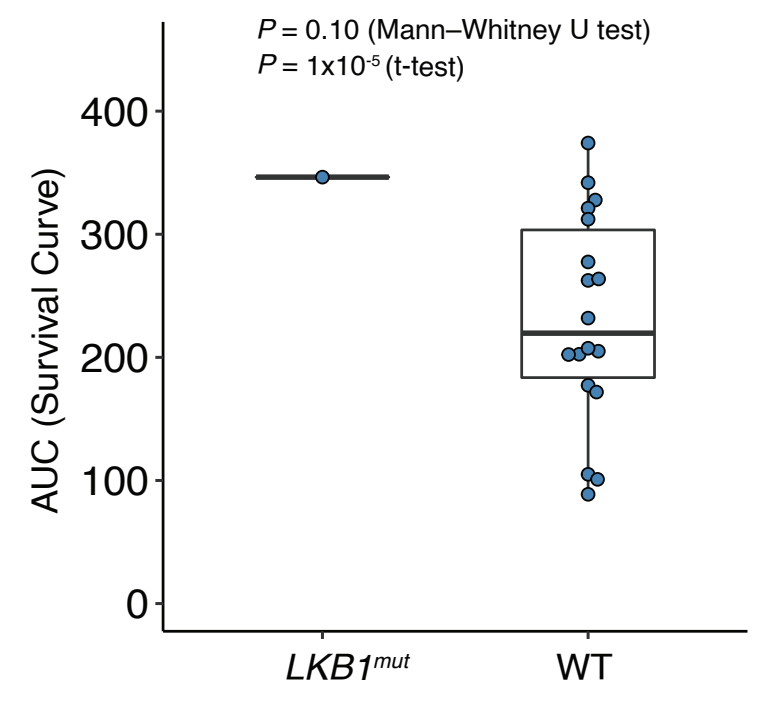

C

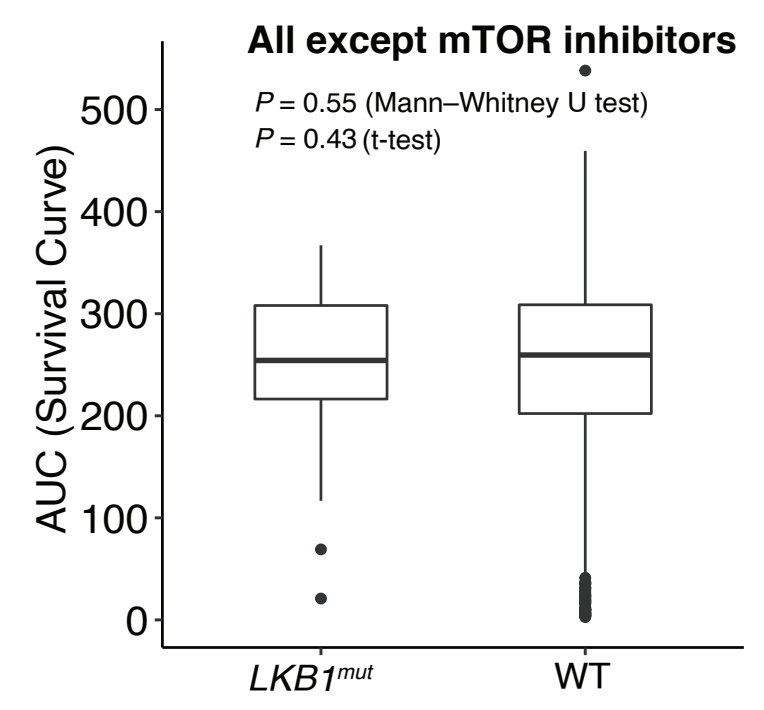

b

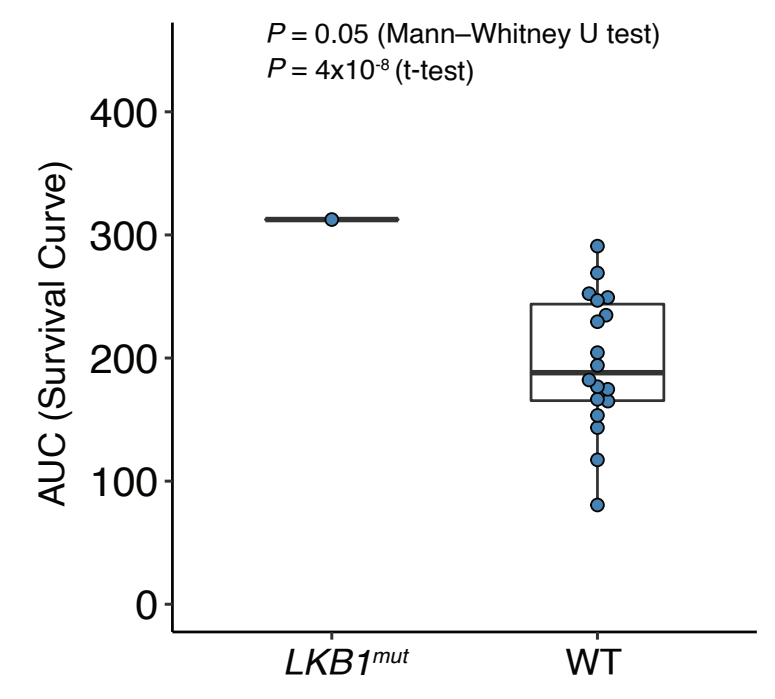

d

\footnotetext{
Supplementary Figure 13. Correlation of $L K B 1$-mutation with resistance to $\mathrm{mTOR}$ inhibition in patient-derived cancer cells from patients with lung adenocarcinoma.

a,b. Jin-Ku Lee et al. quantified the area under the curve (AUC) of the dose-response curves as a proxy for the pharmacological responses of patient-derived cancer cells from 462 patients to 60 treatments (the Cancer-Drug eXplorer database). We focused on the 21 primary cell cultures derived from patients with lung adenocarcinomas. Among them, one had an LKB1 mutation and those cells showed resistance to two mTOR inhibitor, everolimus (a), and AZD2014 (b). $P$-values were calculated from one-sided Mann-Whitney U-test or one-sided t-test.

c. The LKB1-mutant tumor-derived cells do not show general resistance to treatments. Another explanation for the resistance of that one sample to mTOR inhibition could be that those cancer cells (with the LKB1 mutation) are just more resistant to therapies in general. The AUC for $\angle K B 1$ to all treatments other than mTOR inhibitors were not different from cell lines that are wild-type in LKB1. 985 data points were used for comparison and we did not plot individual data points for better visualization.
} 
bioRxiv preprint doi: https://doi.org/10.1101/2020.01.28.923912; this version posted January 29, 2020. The copyright holder for this preprint (which was not certified by peer review) is the author/funder. All rights reserved. No reuse allowed without permission.

Li, Lin et al.

a

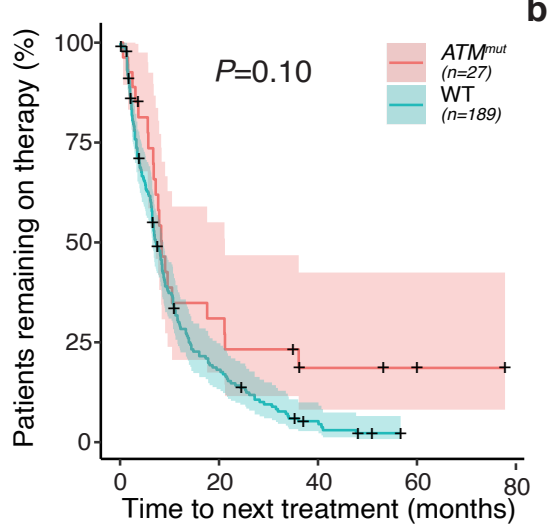

d

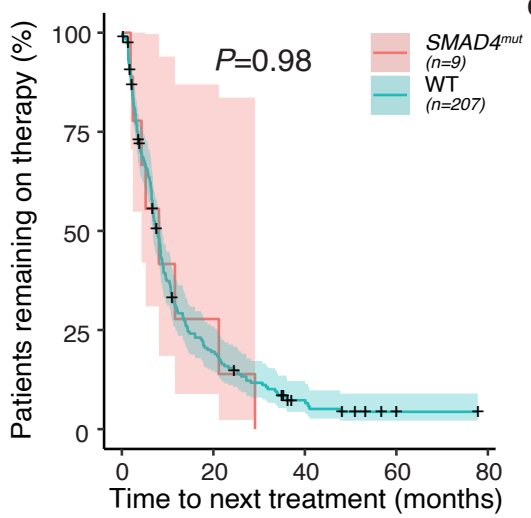

$\mathbf{g}$

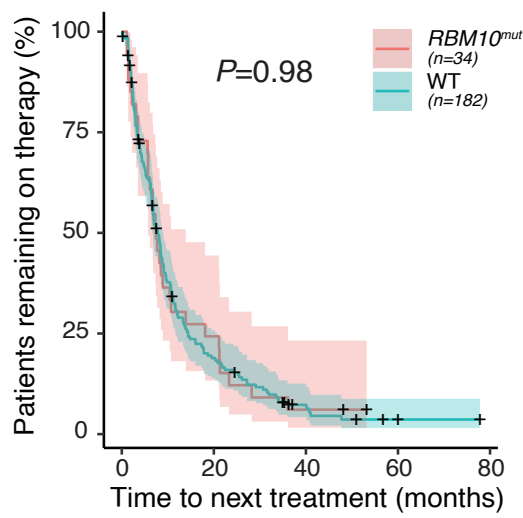

j

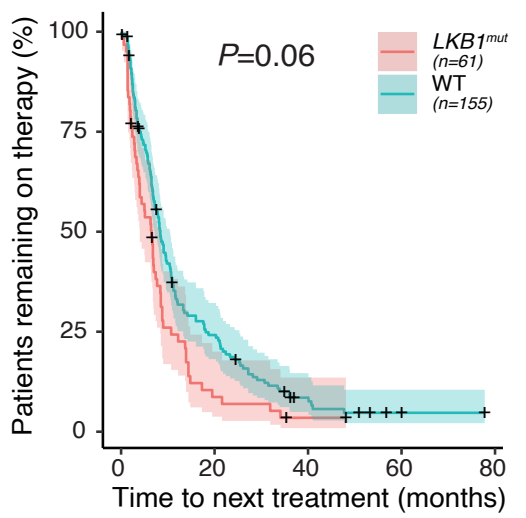

b

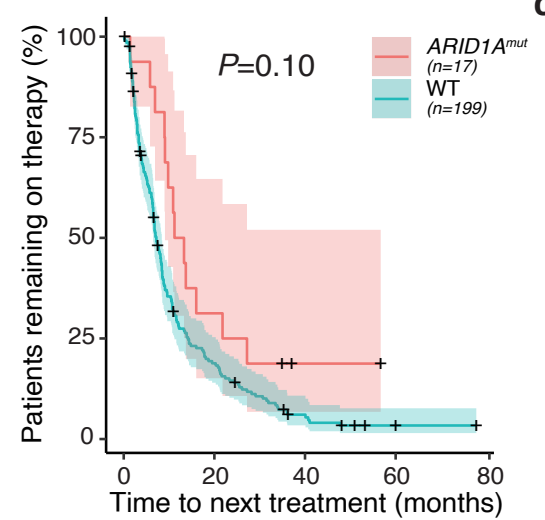

e

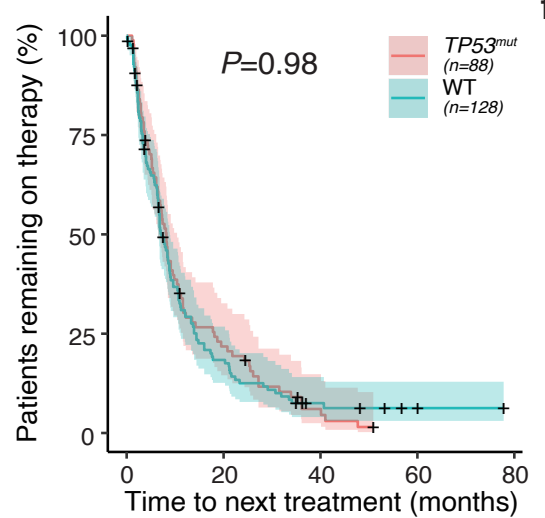

h

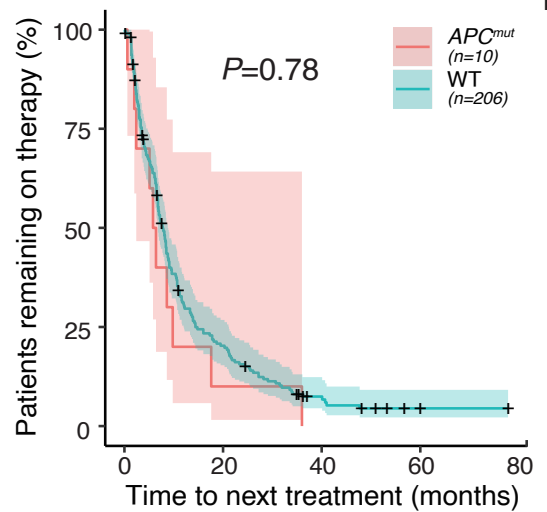

K

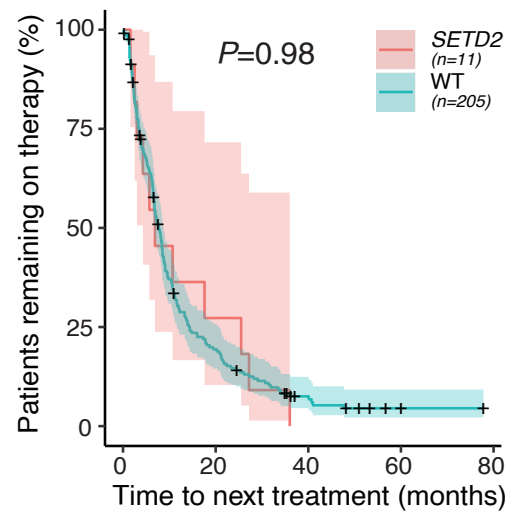

C

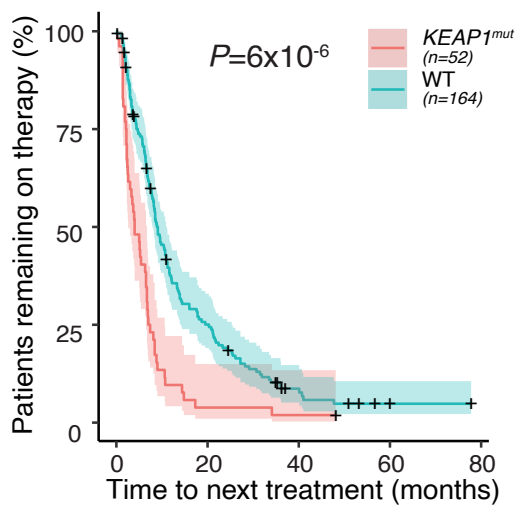

f

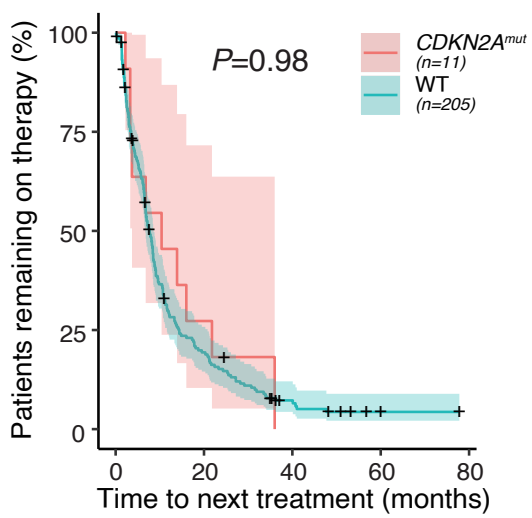

i

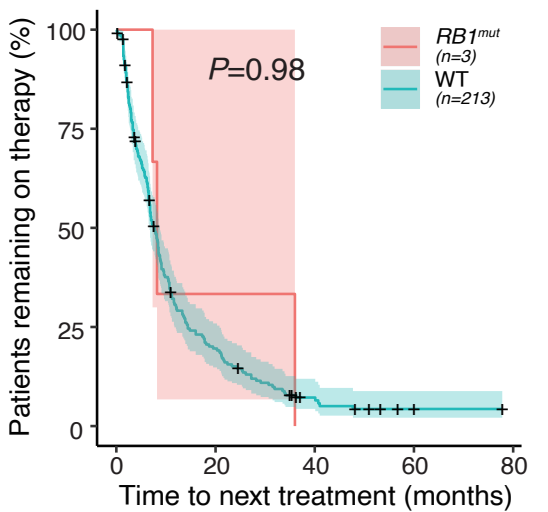

1

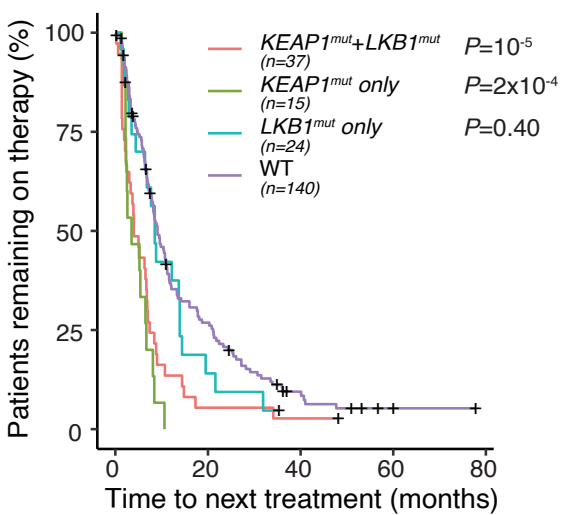

Supplementary Figure 14. Patient response to chemotherapy represented by Kaplan-Meier estimator plot

a-k. Kaplan-Meier curve (with 95\% confidence interval in shading) of time-to-next-treatment (months) for patients with or without mutation of the gene of interest. Longer time-to-next-treatment is associated with better responses to chemotherapy. Here, we analyzed data from a total of 216 patients with mutations in KRAS codon 12,13, or 16. P-values were calculated from the Mantel-Haenszel test with FDR correction. Genotypes compared are indicated for each plot and the number of patients in each group is shown.

I. KEAP1 mutations commonly co-occur with $L K B 1$ mutations. We divide patients into four groups based on their mutation status of $L K B 1$ and $K E A P 1$, and found that the nominal resistance of the LKB1 mutant group (see Figure S13j) seems to be due to the effect of co-incident KEAP1 mutations. Importantly, the absence of resistance conferred by LKB1 mutation is consistent with our mouse model data. $P$-values were calculated relative to WT using the Mantel-Haenszel test with FDR correction. 
bioRxiv preprint doi: https://doi.org/10.1101/2020.01.28.923912; this version posted January 29, 2020. The copyright holder for this preprint (which was not certified by peer review) is the author/funder. All rights reserved. No reuse allowed without permission.

$\mathrm{Li}$, Lin et al.

\section{Identification of GSTR that results in tumors $50 \%$ larger than expected $(G=+50 \%)$}

\section{a}

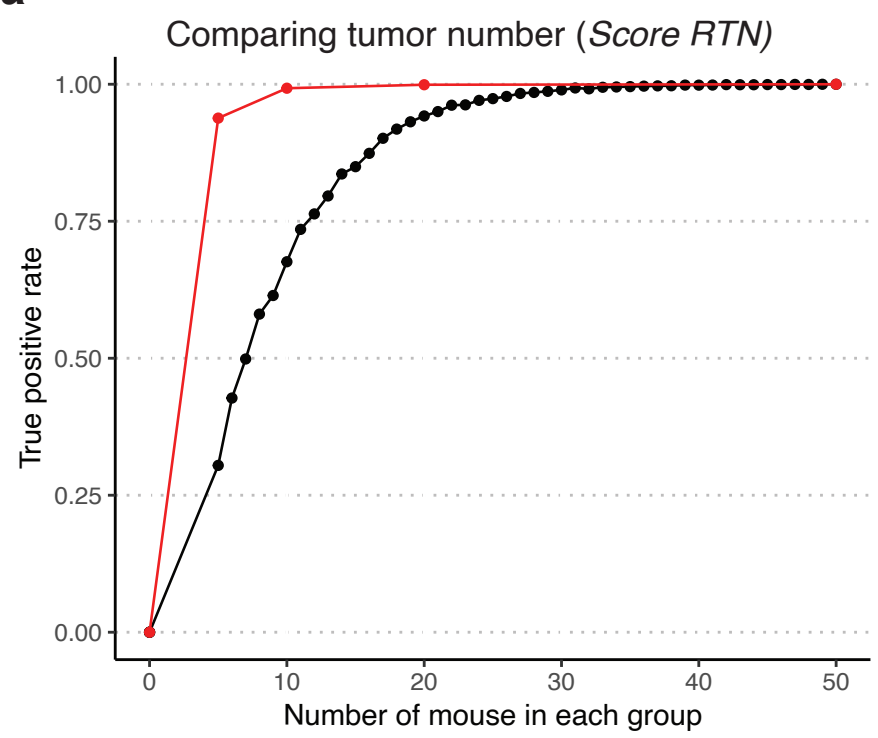

True positive rate when using 5 mice 94\% (our approach) $32 \%$ (one vector per mouse)

b

Comparing geometric mean (Score RGM)

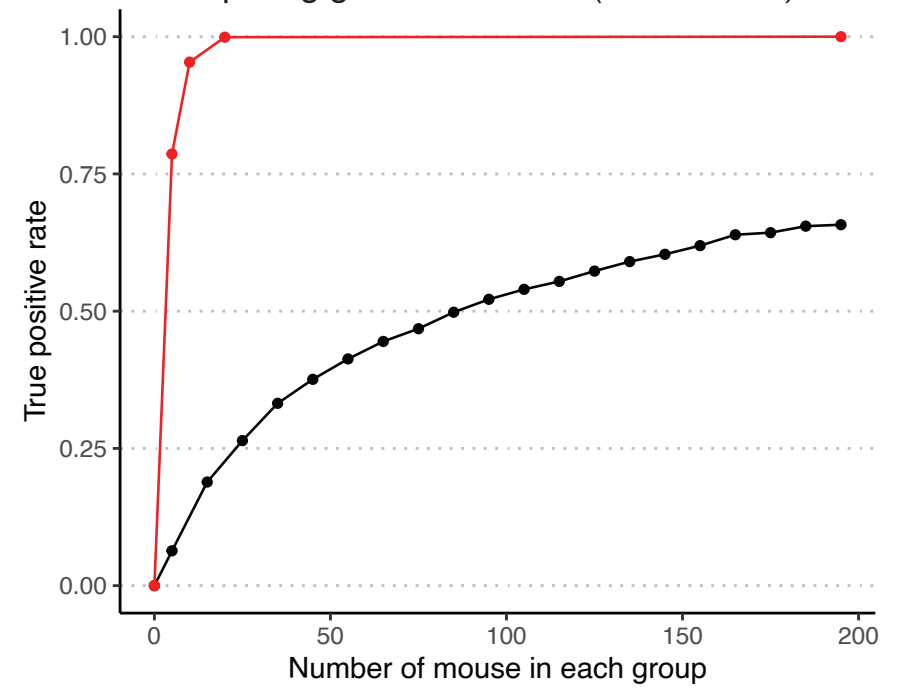

True positive rate when using 5 mice $79 \%$ (our approach) $6 \%$ (one vector per mouse)

Supplementary Figure 15. The Tuba-seq platform greatly reduces the amount of work and costs for identifying genotype-specific therapeutic responses.

a. The sensitivity of using our Tuba-seq method (ScoreRTN metric) with multiplexed vectors is higher than having one vector per mouse and comparing the number (and/or size) of tumors between untreated and treated cohorts. We performed 1000 simulations for each sample size and calculated the probability of identifying the preassigned GSTR. In our study, using five mice in each group has a high true positive rate of $94 \%$ for identifying GSTRs with $G=+50 \%$. For the one vector per mouse approach, to achieve only a $50 \%$ true positive rate, we would need $\sim 7$ mice per vector, and a total of 672 mice ( 7 mice for each of the 12 genotypes under eight treatments). The cost of doing the study with one genotype per mouse would be 17-times the cost to achieve half of our true positive rate. Keep in mind that this includes a 17-fold increase in the cost of animal housing, 17-fold increase in viral vector production, 17-fold increase in the amount of each drug need, 17-fold increase in the number of mouse dosings, and 17-fold increase in the number of Tuba-seq libraries and sequencing costs. Labor-related costs would also be much higher, and the total cost of a one genotype per mouse study would exceed several million dollars. Following the same logic, to achieve a similar true positive rate of $94 \%, \sim 50$ times more mice would be needed with proportional increases in all costs.

b. Sensitivity for using our Tuba-seq method (ScoreRGM metric) with multiplexed vectors is much higher than having one vector per mouse and comparing the geometric mean of tumors between the untreated and treated mice. Note that assuming uniform GSTR, the metric that compares the tumor numbers has higher statistical power than the metric comparing the geometric means, and the x-axes are plotted on different scales for better visualization. Using the size metric ScoreRGM alone, having five mice in each group results in a true positive rate of $79 \%$ in identifying GSTR with $G=+50 \%$. For the one vector per mouse approach, to achieve a $50 \%$ true positive rate, we would need $\sim 85$ mice per vector, which is a total of 8160 mice for performing the pharmacogenomic mapping experiment. The total cost for a study like that would be astronomical. 